\title{
A proposed baroclinic wave test case for deep- and shallow-atmosphere dynamical cores
}

\author{
Paul A. Ullrich, ${ }^{\text {a }}$ Thomas Melvin, ${ }^{\mathrm{b}}$ Christiane Jablonowski ${ }^{\mathrm{c}}$ and Andrew Staniforth ${ }^{\mathrm{b}}$ \\ ${ }^{a}$ Department of Land, Air and Water Resources, University of California, Davis, CA, USA \\ ${ }^{\mathrm{b}}$ Met Office, Exeter, UK \\ ${ }^{c}$ Department of Atmospheric, Oceanic and Space Sciences, University of Michigan, Ann Arbor, MI, USA
}

${ }^{*}$ Correspondence to: P. Ullrich, Department of Land, Air and Water Resources, University of California, One Shields Avenue, Davis, CA 95616, USA. E-mail: paullrich@ucdavis.edu

This article is published with the permission of the Controller of HMSO and the Queen's Printer for Scotland.

Idealised studies of key dynamical features of the atmosphere provide insight into the behaviour of atmospheric models. A very important, well understood, aspect of midlatitude dynamics is baroclinic instability. This can be idealised by perturbing a vertically sheared basic state in geostrophic and hydrostatic balance. An unstable wave mode then results with exponential growth (due to linear dynamics) in time until, eventually, nonlinear effects dominate and the wave breaks.

A new, unified, idealised baroclinic instability test case is proposed. This improves on previous ones in three ways. First, it is suitable for both deep- and shallow-atmosphere models. Second, the constant surface pressure and zero surface geopotential of the basic state makes it particularly well-suited for models employing a pressure- or height-based vertical coordinate. Third, the wave triggering mechanism selectively perturbs the rotational component of the flow; this, together with a vertical tapering, significantly improves dynamic balance.

Key Words: baroclinic instability; model intercomparison; small Earth

Received 26 November 2012; Revised 20 August 2013; Accepted 25 August 2013; Published online in Wiley Online Library 2 December 2013

\section{Introduction}

Exact solutions of the governing equations of the atmosphere are very useful for the development and testing of numerical models (Williamson et al., 1992; Läuter et al., 2005; Staniforth and White, 2007, 2008a,b; White and Staniforth, 2008, 2009). Most known exact solutions are steady, with a dearth of timedependent solutions to test the important temporal behaviour of numerical models. Exceptionally, the Läuter et al. (2005) and Staniforth and White (2008b) solutions are unsteady. However they are based on the transformation of steady solutions in an inertial frame of reference to a rotating frame of reference, leading to periodic solutions of intrinsic diurnal frequency. None of these exact solutions represent a very important property of the governing equations, viz. baroclinic development. To this end, and inspired by the work of Hoskins and Simmons (1975) and Simmons and Hoskins (1975), Jablonowski and Williamson (2006a) (hereafter referred to as JW06) and Jablonowski and Williamson (2006b) proposed a baroclinic instability test case for atmospheric dynamical cores.

Their proposal consists of two parts. First, a model is initialised with a balanced steady-state solution of the hydrostatic primitive equations which is stable to axisymmetric disturbances about the Earth's rotation axis. This tests the extent to which a model can maintain this exact solution in the presence of numerical truncation and round-off errors. Second, a small-amplitude, relatively large-scale, but localised, Gaussian hill perturbation of the zonal wind field in northern midlatitudes is superimposed on this atmospheric state at initial time. This then triggers the evolution of a baroclinic wave over the course of several days. Since, for this part of the test, no analytic solution exists, an accurate approximation to the exact solution is obtained using an ensemble of high-resolution reference numerical solutions. The JW06 test case has proven to be very useful. For example, it enabled Williamson et al. (2009) to expose and remedy two dynamical core formulation flaws.

In a related development, Lauritzen et al. (2010) suggest rotating the computational grid of the JW06 test case so that the balanced flow is no longer aligned with a latitude-longitude (lat-lon) grid, thereby eliminating a favourable bias of the JW06 test case towards models based on lat-lon grids. Lauritzen et al. (2010) then used this modified test case to examine the performance of six dynamical cores which employ different computational grids (regular lat-lon, cubed-sphere, icosahedral hexagonal/triangular) and different numerical schemes. The results of all models agreed, to within an acceptable tolerance, with one another at sufficiently high resolution, confirming the value of an ensemble of high-resolution integrations as a proxy for the exact solution. Of further interest is the degree of 'grid imprinting' observed as a function of model grid and 
associated discretisation, i.e. the extent to which the geometry of an underlying computational grid affects the structure of the numerical solution and the geographic distribution of errors. (Errors are typically largest, and systematic, in the vicinity of any special points of the underlying geometry: e.g. around the poles of a lat-lon grid, and the vertices of sphered cubes and icosahedra.)

The basic state of the JW06 test problem is constructed to be an exact steady axially symmetric state of the hydrostatic primitive equations. Because the vertical acceleration, $\mathrm{D} w / \mathrm{D} t$, is identically zero for this basic state, it is also an exact steady state of the non-hydrostatic primitive equations. ${ }^{1}$

Although JW06's basic state is an exact steady state of the shallow-atmosphere equations, it is not an exact steady state of the deep-atmosphere equations. However, with the advent of evermore-powerful computers, numerical atmospheric models based on the deep-atmosphere equations have become operationally feasible (Cullen, 1993; Davies et al., 2005), and the development of deep-atmosphere dynamical cores is currently an active area of research (Staniforth and Wood, 2008; Satoh et al., 2008; Walko and Avissar, 2008; Ullrich and Jablonowski, 2012a; Wood et al., 2013). Therefore there is growing interest in devising suitable test problems to aid in the validation of deep-atmosphere dynamical cores. The question thus arises as to how to construct an analogous baroclinic-wave test case to the JW06 one, which is appropriate not only for shallow-atmosphere dynamical cores, but also for deep-atmosphere ones. The goal of this work is to provide an answer to this question. Although some sample illustrative results from model integrations of several models are presented herein, an in-depth intercomparison of model performance for the new test case, similar to that given in JW06 for their more-limited test case, is beyond the scope of the present study.

It is highly desirable that basic-state fields, and initial perturbations, be expressed in an analytical manner. The very essence of the problem is thus to devise an appropriate exact steady axially symmetric basic-state solution of the deep-atmosphere equations which, when suitably perturbed, captures the essential features of a baroclinic wave, and to do so in such a way that it straightforwardly leads to an analogous shallow-atmosphere basic state which behaves similarly when similarly perturbed. This is not at all as easy as one might expect. An obvious approach is to follow the JW06 derivation for a shallow atmosphere, but instead to use the deep-atmosphere equations. This was attempted, but proved fruitless. The JW06 derivation is intrinsically based on the use of an isobaric coordinate system, and it leads to $r$ (spherical radius) varying along isobaric surfaces. Unfortunately, many terms in the deep-atmosphere equations have an explicit functional dependence on $r$ which is missing in the shallowatmosphere equations: application of the shallow-atmosphere assumption results in $r$ being systematically replaced by $a$ (Earth's radius), wherever it appears as a coefficient. This, coupled with the existence of additional (Coriolis and metric) terms, and consequent different (and more complex) horizontal and vertical balances, renders the problem analytically intractable in isobaric coordinates. A key element of the approach taken herein is therefore to abandon the use of the isobaric coordinate system, and instead to adopt spherical radius (and/or its equivalent, geometric height, defined by $z \equiv r-a$ ) as vertical coordinate. It is then possible to exploit the exact shallow- and deep-atmosphere class of steady axisymmetric solutions derived in Staniforth and White (2011) and Staniforth and Wood (2013) (hereafter referred to as SW11 and SW12, respectively).

JW06 use a mean temperature profile which is constructed by adding a term, above the tropopause, to a background profile: this term then leads to the representation of an idealised stratosphere. However, consistent with synoptic baroclinic development being a

\footnotetext{
${ }^{1}$ The reader unfamiliar with the definition of the various equation sets discussed herein, and the key differences between them, is referred to the in-depth discussion of White et al. (2005), and to the summary discussion given in section 2 herein.
}

tropospheric phenomenon, it has been found by experimentation (C. Smith and J. Thuburn, 2011; personal communication) that omitting this term has virtually no impact on the development of the baroclinic wave. Thus no attempt is made to include a realistic representation of the stratosphere in the basic state for the test problem proposed herein.

The organisation of the article is as follows. The nonhydrostatic deep-atmosphere equations are given in section 2, together with a discussion of three related equation sets. The deep-atmosphere basic state for the test problem is defined in section 3 , the analogous shallow-atmosphere one in section 4 , and the baroclinic wave-triggering mechanism in section 5. Details of the configuration of the basic state for the test problem, including parameter values, are given in section 6. Illustrative results using various models may be found in section 7 , and conclusions are drawn in section 8 .

\section{Governing equations}

In standard notation, the governing equations for a dry deep spherical atmosphere are (White et al., 2005; White and Staniforth, 2008):

$$
\begin{aligned}
\frac{\mathrm{D} u}{\mathrm{D} t}+\frac{u w}{r}- & \frac{u v \tan \phi}{r} \\
& +2 \Omega w \cos \phi-2 \Omega v \sin \phi+\frac{R T}{r \cos \phi} \frac{\partial q}{\partial \lambda}=0, \\
\frac{\mathrm{D} v}{\mathrm{D} t}+\frac{v w}{r}+\frac{u^{2} \tan \phi}{r}+2 \Omega u \sin \phi+\frac{R T}{r} \frac{\partial q}{\partial \phi} & =0, \\
\frac{\mathrm{D} w}{\mathrm{D} t}-\frac{\left(u^{2}+v^{2}\right)}{r}-2 \Omega u \cos \phi+g\left(\frac{a}{r}\right)^{2}+R T \frac{\partial q}{\partial r}=0, & \frac{\mathrm{D} \theta}{\mathrm{D} t}=0, \quad p=\rho R T .
\end{aligned}
$$

Here

$$
\begin{gathered}
q \equiv \ln \left(\frac{p}{p_{00}}\right), \quad \theta \equiv\left(\frac{p}{p_{00}}\right)^{-\kappa} T, \\
u \equiv r \cos \phi \frac{\mathrm{D} \lambda}{\mathrm{D} t}, \quad v \equiv r \frac{\mathrm{D} \phi}{\mathrm{D} t}, \quad w \equiv \frac{\mathrm{D} r}{\mathrm{D} t}, \\
\mathcal{D} \equiv \frac{1}{r \cos \phi} \frac{\partial u}{\partial \lambda}+\frac{1}{r \cos \phi} \frac{\partial}{\partial \phi}(v \cos \phi)+\frac{1}{r^{2}} \frac{\partial}{\partial r}\left(r^{2} w\right), \\
\frac{\mathrm{D}}{\mathrm{D} t} \equiv \frac{\partial}{\partial t}+\frac{u}{r \cos \phi} \frac{\partial}{\partial \lambda}+\frac{v}{r} \frac{\partial}{\partial \phi}+w \frac{\partial}{\partial r},
\end{gathered}
$$

$p_{00}$ being a constant reference pressure.

Equations (1)-(3) are, respectively, the longitudinal $(\lambda)$, latitudinal $(\phi)$ and radial $(r)$ components of the momentum equation. Equations (4) are the continuity equation, the thermodynamic equation, and the ideal gas law. The gravity term in the radial momentum equation (3) varies as $r^{-2}$ so as to preserve basic kinematic and geometric properties under the spherical geopotential approximation (White et al., 2005); $g$ is a representative mean value of the acceleration due to gravity at the Earth's surface, and $a$ is the Earth's mean radius.

A very brief overview is now given regarding the definition of the four closely related equation sets discussed in depth in White et al. (2005), and the nomenclature used to refer to them herein. The most general of these are Eqs (1)-(8), and they are termed the non-hydrostatic deep-atmosphere equations. Dropping the vertical acceleration term $\mathrm{D} w / \mathrm{D} t$ from the vertical momentum equation (3) gives the quasi-hydrostatic deep-atmosphere equations. (Quasihydrostatic, as opposed to hydrostatic, is used to reflect the fact that when $\mathrm{D} w / \mathrm{D} t \equiv 0$, the force balance in the deep-atmosphere vertical momentum equation is not exactly hydrostatic: this is 
due to the presence of the metric terms $-\left(u^{2}+v^{2}\right) / r$ and of the Coriolis term $-2 \Omega u \cos \phi$, and also to the presence of the vertically varying factor $(a / r)^{2}$ in the term for the acceleration due to gravity, all of which are neglected when making the hydrostatic approximation.)

There are two analogous shallow-atmosphere equation sets. For both of these, the shallow-atmosphere assumption is made, whereby

- $r$ is replaced everywhere in Eqs (1)-(8) by $a$, except where it appears as $\partial / \partial r$, on the basis that the depth of the Earth's atmosphere is two orders of magnitude smaller than $a$.

Also the so-called 'traditional' assumptions are made (these are needed to preserve the underlying conservation properties on which the governing equations are based; White et al. (2005)) whereby

- the $2 \Omega \cos \phi$ Coriolis terms are dropped from Eqs (1) and (3); and

- the metric terms $u w / r, v w / r$ and $-\left(u^{2}+v^{2}\right) / r$ are dropped from Eqs (1), (2) and (3), respectively.

The only difference between the two shallow-atmosphere equation sets is the absence or presence of the vertical acceleration term $\mathrm{D} w / \mathrm{D} t$ in the resulting shallow-atmosphere vertical momentum equation

$$
\frac{\mathrm{D} w}{\mathrm{D} t}+g+R T \frac{\partial q}{\partial r}=0 .
$$

In the absence of $\mathrm{D} w / \mathrm{D} t$, Eq. (9) reduces to hydrostatic balance and, together with the other modified equations, the well-known (shallow-atmosphere) hydrostatic primitive equations result. These are the shallow-atmosphere analogue of the quasihydrostatic deep-atmosphere equations. Equation (9) (with Dw/Dt retained), together with the other modified equations, results in the (shallow-atmosphere) non-hydrostatic primitive equations, which are the shallow-atmosphere analogue of the the nonhydrostatic deep-atmosphere equations.

It is well known that synoptic-scale, midlatitude, tropospheric flows, such as baroclinic instability, are both qualitatively and quantitatively well represented by any of the above-described equation sets, the most approximated of which is the hydrostatic primitive equation set. This is because, for these flows, the shallowatmosphere and hydrostatic approximations are very accurate, and dropped terms are insignificant. (If this were not so, then operational medium-range weather forecast models based on the hydrostatic primitive equations, i.e. most current operational models, would be very poor, which is not the case.) Thus, for the baroclinic-wave test problem described herein (with parameters set at Earth values), one expects that integrations performed with any of these equations sets should give solutions that are qualitatively and quantitatively very close to one another. For the small-Earth experiments discussed in section 7.3, this is no longer the situation: the reduction of Earth's (true) radius by a factor of twenty is such that the shallow-atmosphere assumption no longer holds, and deep- and shallow-atmosphere results can then be expected to differ significantly from one another.

\section{Deep-atmosphere basic state}

\subsection{A class of exact solutions}

A broad class of closed-form non-separable exact zonal solutions to the nonlinear equations (1)-(8) is developed in SW11 and SW12. These solutions are exploited herein to define the basic state for the proposed baroclinic-wave test problem. To obtain an exact nonlinear midlatitude baroclinic jet solution to Eqs (1)-(8), SW11 sought axially symmetric solutions of the form

$$
\begin{array}{r}
u=u(\phi, r), \quad v=w=0, \\
\rho=\rho(\phi, r), \quad T=T(\phi, r), \quad p=p(\phi, r) .
\end{array}
$$

This leads to a set of trivially satisfied equations plus the two non-trivial constraint equations (cf. Eqs (2) and (3) of SW11):

$$
\begin{aligned}
\frac{u^{2}}{r} \tan \phi+2 \Omega u \sin \phi+\frac{R T}{r} \frac{\partial \ln p}{\partial \phi} & =0, \\
-\frac{u^{2}}{r}-2 \Omega u \cos \phi+g \frac{a^{2}}{r^{2}}+R T \frac{\partial \ln p}{\partial r} & =0 .
\end{aligned}
$$

SW11 assumed (their Eq. (8)) a temperature field of a form equivalent to

$$
\begin{aligned}
& T(\phi, r)=\left(\frac{a}{r}\right)^{2} \\
& \quad \times\left[\tilde{\tau}_{1}(r)-\tilde{\tau}_{2}(r)\left\{\left(\frac{r}{a} \cos \phi\right)^{k}-\left(\frac{k}{k+2}\right)\left(\frac{r}{a} \cos \phi\right)^{k+2}\right\}\right]^{-1},
\end{aligned}
$$

where $\tilde{\tau}_{1}(r)$ and $\tilde{\tau}_{2}(r)$ are fairly arbitrary functions, constrained by atmospheric realism and tractable integration, and $k \geq 2$ is assumed integer. (The precise connection between Eq. (13) above, and SW11's Eq. (8), is that $\widetilde{\tau}_{1}(r) \equiv(r / a) \tau_{1}(r)$ and $\tilde{\tau}_{2}(r) \equiv(r / a) \tau_{2}(r)$. Rewriting the functional form of $T$ in this way then simplifies the parallel treatment of the deepand shallow-atmosphere cases.) They then derived the balancing flow and accompanying pressure field: cf. their Eqs (12), (13) and (24). The SW11 solutions are analytic, provided that the functional forms of $\widetilde{\tau}_{1}(r)$ and $\widetilde{\tau}_{2}(r)$ are chosen such that the integrals $\int_{a}^{r} \tilde{\tau}_{1}\left(r^{\prime}\right) \mathrm{d} r^{\prime}$ and $\int_{a}^{r} \tilde{\tau}_{2}\left(r^{\prime}\right) \mathrm{d} r^{\prime}$ may be obtained in closed form. The reciprocal functional form of $T(\phi, r)$ in Eq. (13) was adopted in SW11 because $T$ appears as $1 / T$ in the compatibility equation (their Eq. (4)), which is the basis for their derivation of a class of exact solutions. The factor $(a / r)^{2}$, which would otherwise be absent, is due to the inclusion in Eqs (3) and (12) of vertical variation of gravity.

3.2. The basic state

Let

$$
\begin{aligned}
\tilde{\tau}_{1}(r)= & A \frac{\Gamma}{T_{0}} \exp \left\{\frac{\Gamma}{T_{0}}(r-a)\right\} \\
& +B\left\{1-2\left(\frac{r-a}{b H}\right)^{2}\right\} \exp \left\{-\left(\frac{r-a}{b H}\right)^{2}\right\}, \\
\tilde{\tau}_{2}(r)= & C\left\{1-2\left(\frac{r-a}{b H}\right)^{2}\right\} \exp \left\{-\left(\frac{r-a}{b H}\right)^{2}\right\},
\end{aligned}
$$

where $T_{0}$ is a representative constant value of temperature, $\Gamma$ is an assumed lapse rate, $H \equiv R T_{0} / g$ is the scale height of the atmosphere, $b$ is a half-width parameter, and $A, B$ and $C$ are arbitrary constant parameters.

These functional forms for $\widetilde{\tau}_{1}(r)$ and $\widetilde{\tau}_{2}(r)$ are inspired by those used in Staniforth (2012) for exact solutions in $z$ coordinates on a $\beta-\gamma$ plane, which were in turn inspired by those used in Ullrich and Jablonowski (2012b) for their channel flow test problems in isobaric coordinates on a $\beta$ plane. They also correspond to the illustrative example solution examined in section 3.1 of SW12 in the context of a broader class of exact solutions of the Euler equations.

As shown in SW12, $A, B$ and $C$ can be equivalently rewritten in terms of more meaningful physical quantities. Consider the special case $B=C=0$, i.e. when (from Eqs (13)-(15)) the temperature only varies vertically, but not horizontally. Evaluating Eq. (13) at $r=a$, with $B=C=0$ in Eqs (14) and (15), then gives $T(r=a)=T_{0} /(A \Gamma)$. It is therefore natural to set

$$
A=\frac{1}{\Gamma}
$$


and $T_{0}$ is then to be interpreted as being a representative surface value of temperature, in the absence of horizontally varying perturbations. The physical interpretation of $\Gamma$ now becomes apparent by: setting $A=1 / \Gamma$ and $B=C=0$ in Eqs (14) and (15); using these in Eq. (13); and differentiating the resulting equation with respect to $r$, followed by evaluation at $r=a$. This then yields $\left.(\mathrm{d} T / \mathrm{d} r)\right|_{r=a}=-\Gamma$. Thus $\Gamma$ can be interpreted as being a representative value of the lapse rate of temperature at $r=a$, as in JW06.

Evaluating Eq. (13) at $r=a$, and then at the Equator and at the two Poles, i.e. at $\phi=0, \pm \pi / 2$, allows the two arbitrary parameters $B$ and $C$ to be expressed in terms of the two more meaningful physical quantities $T_{0}^{\mathrm{E}} \equiv T(\phi=0, r=a)$ and $T_{0}^{\mathrm{P}} \equiv T(\phi= \pm \pi / 2, r=a)$, where $T_{0}^{\mathrm{E}}$ and $T_{0}^{\mathrm{P}}$ are the surface values of temperature at the Equator and at the Poles. Thus

$$
B=\frac{T_{0}-T_{0}^{\mathrm{P}}}{T_{0} T_{0}^{\mathrm{P}}}, \quad C=\left(\frac{k+2}{2}\right)\left(\frac{T_{0}^{\mathrm{E}}-T_{0}^{\mathrm{P}}}{T_{0}^{\mathrm{E}} T_{0}^{\mathrm{P}}}\right) .
$$

Although $T_{0}$ can be arbitrarily set to any representative value, for simplicity it is set to

$$
T_{0}=\frac{1}{2}\left(T_{0}^{\mathrm{E}}+T_{0}^{\mathrm{P}}\right)
$$

as in SW12. Using this value in Eq. (17) then gives $B$ and $C$ in terms of the surface temperature values $T_{0}^{\mathrm{E}}$ and $T_{0}^{\mathrm{P}}$ as

$$
B=\frac{\left(T_{0}^{\mathrm{E}}-T_{0}^{\mathrm{P}}\right)}{\left(T_{0}^{\mathrm{E}}+T_{0}^{\mathrm{P}}\right) T_{0}^{\mathrm{P}}}, \quad C=\left(\frac{k+2}{2}\right)\left(\frac{T_{0}^{\mathrm{E}}-T_{0}^{\mathrm{P}}}{T_{0}^{\mathrm{E}} T_{0}^{\mathrm{P}}}\right),
$$

cf. Eq. (46) of SW12.

The arbitrary integration function $F(r \cos \phi)$ in Eq. (12) of SW11 is chosen to be identically zero. This is not only simple, it has two further useful properties. First, it leads to $u$ being identically zero at the surface $r=a$. Second, it makes the isobaric surface

\begin{tabular}{|c|c|c|c|}
\hline Parameter & Value & Unit & Description \\
\hline$a$ & $6.371229 \times 10^{6}$ & $\mathrm{~m}$ & Mean radius of Earth \\
\hline$b$ & 2 & - & Half-width parameter \\
\hline$d_{0}$ & $a / 6$ & - & $\begin{array}{l}\text { Horizontal radius of perturba- } \\
\text { tion domain }\end{array}$ \\
\hline$g$ & 9.80616 & $\mathrm{~ms}^{-2}$ & $\begin{array}{l}\text { Gravitational acceleration at } \\
\text { Earth's surface }\end{array}$ \\
\hline$k$ & 3 & - & $\begin{array}{l}\text { Power used for temperature } \\
\text { field }\end{array}$ \\
\hline$p_{0}$ & $10^{5}$ & $\mathrm{~Pa}$ & Surface pressure \\
\hline$R$ & 287.0 & $\mathrm{~J} \mathrm{~kg}^{-1} \mathrm{~K}^{-1}$ & Gas constant \\
\hline$T_{0}^{\mathrm{E}}$ & 310 & K & Surface equatorial temperature \\
\hline$T_{0}^{\mathrm{P}}$ & 240 & K & Surface polar temperature \\
\hline$V_{\mathrm{p}}$ & 1.0 & $\mathrm{~ms}^{-1}$ & Perturbed wind amplitude \\
\hline$z_{\mathrm{t}}$ & $1.5 \times 10^{4}$ & $\mathrm{~m}$ & Top of perturbation domain \\
\hline$\Gamma$ & 0.005 & $\mathrm{Km}^{-1}$ & Lapse rate \\
\hline$\left(\lambda_{c}, \phi_{c}\right)$ & $(\pi / 9,2 \pi / 9)$ & - & $\begin{array}{l}\text { Geographical location of per- } \\
\text { turbation centre }\end{array}$ \\
\hline$\Omega$ & $7.29212 \times 10^{-5}$ & $s^{-1}$ & Earth's angular velocity \\
\hline
\end{tabular}
$p=p_{0}$ coincide with the surface $r=a$. This is very convenient
Table 1. Parameter values.

for setting up the test problem developed herein, since the lower surface is then a coordinate surface for both height-based and pressure-based coordinates (this is not so for the JW06 basic state). In pressure-based coordinates this choice is equivalent to setting the surface geopotential to be $0 \mathrm{~m}^{2} \mathrm{~s}^{-2}$ globally.

The proposed deep-atmosphere basic state is summarised in Appendix A.

\section{The analogous shallow-atmosphere basic state}

As shown in SW11 and SW12, it is straightforward to develop a shallow-atmosphere analogue of the deep-atmosphere basic-state solution described above.

The shallow-atmosphere analogue of the deep-atmosphere functional form (13) for $T(\phi, r)$ is obtained by simply setting the factor $(a / r)^{2}$ equal to unity therein. This is because, for
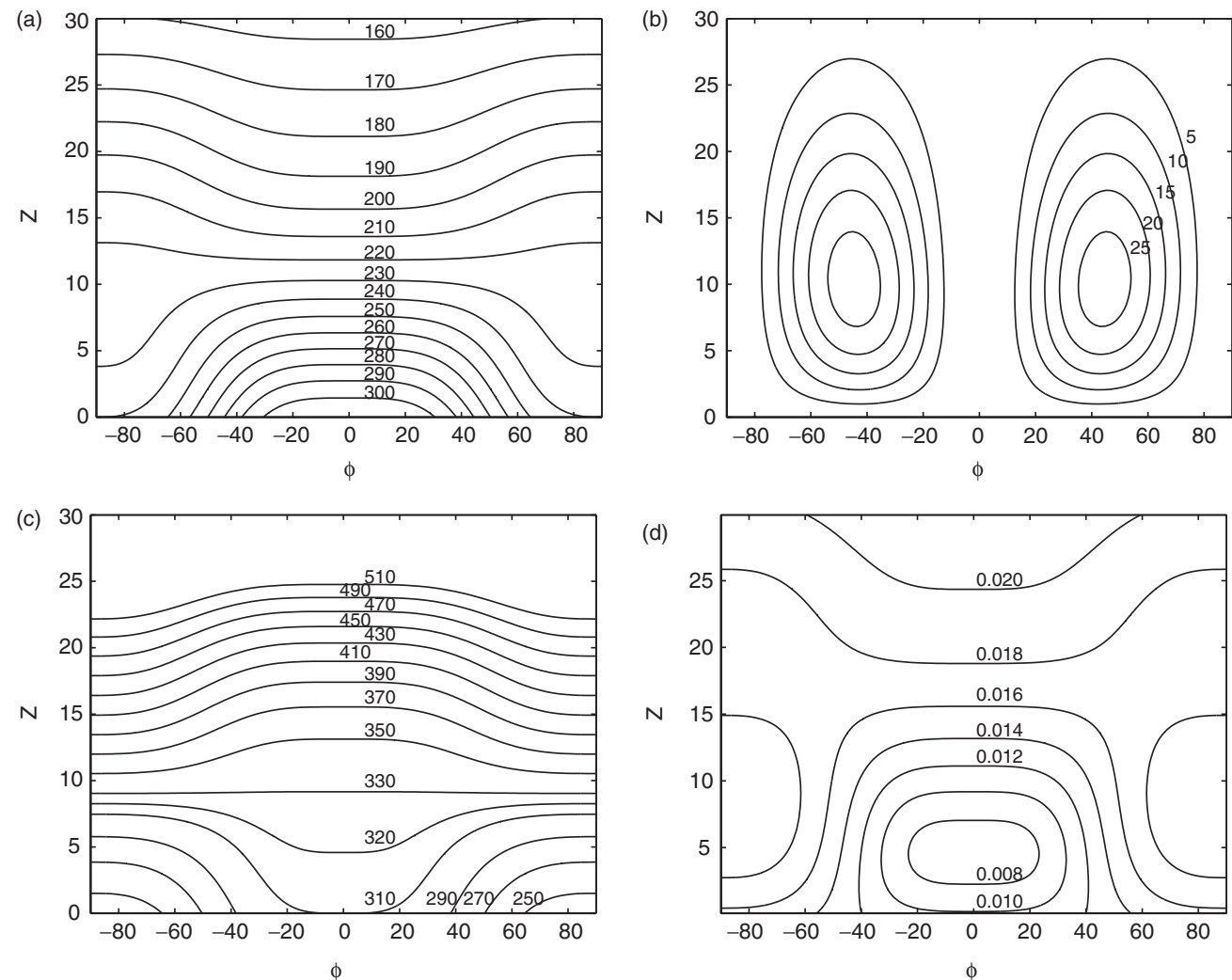

Figure 1. Deep-atmosphere basic-state fields for: (a) temperature $T(\phi, z)$, contour interval $10 \mathrm{~K}$; (b) zonal wind $u(\phi, z)$, contour interval $5 \mathrm{~ms} \mathrm{~s}^{-1}$; (c) potential temperature $\theta(\phi, z)$, contour interval $20 \mathrm{~K}$, but with an additional $320 \mathrm{~K}$ contour; and (d) Brunt-Väisälä frequency $N(\phi, z)$, contour interval $0.002 \mathrm{~s}{ }^{-1}$. Height $z$ is in kilometres. 

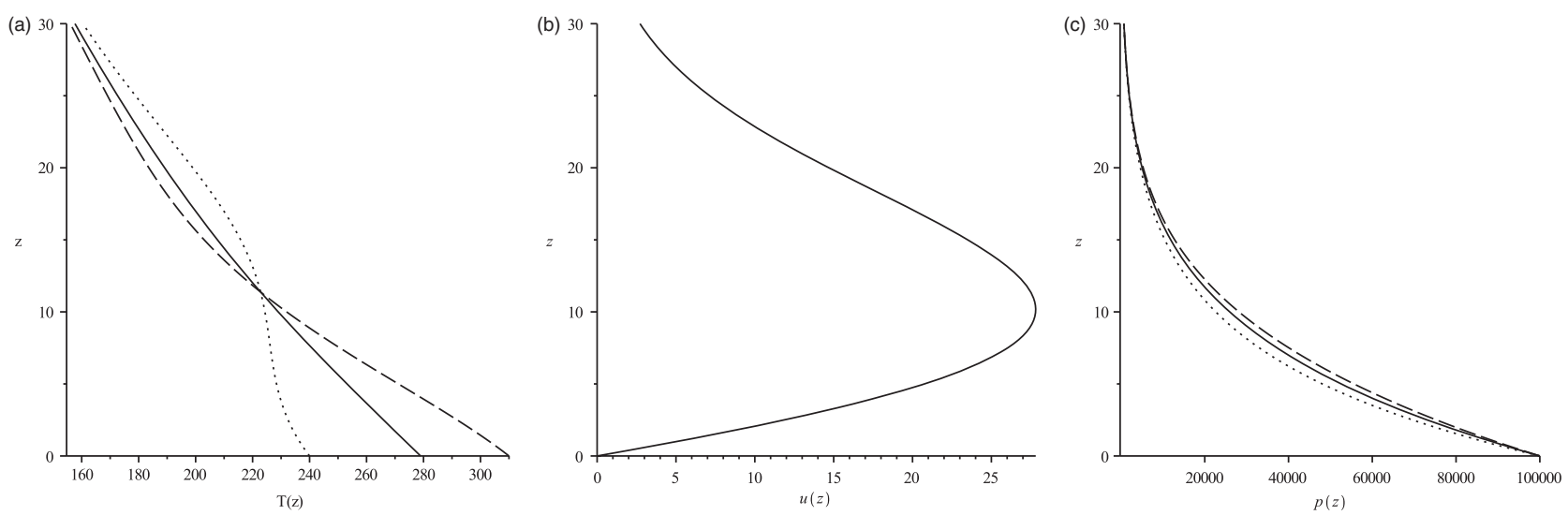

Figure 2. Deep-atmosphere polar (dotted), midlatitude (solid) and equatorial (dashed) profiles for: (a) temperature $T(z)$ in K; (b) zonal wind $u(z)$ in $\mathrm{m} \mathrm{s}^{-1}$; and (c) pressure $p(z)$ in Pa. Height $z$ is in kilometres.
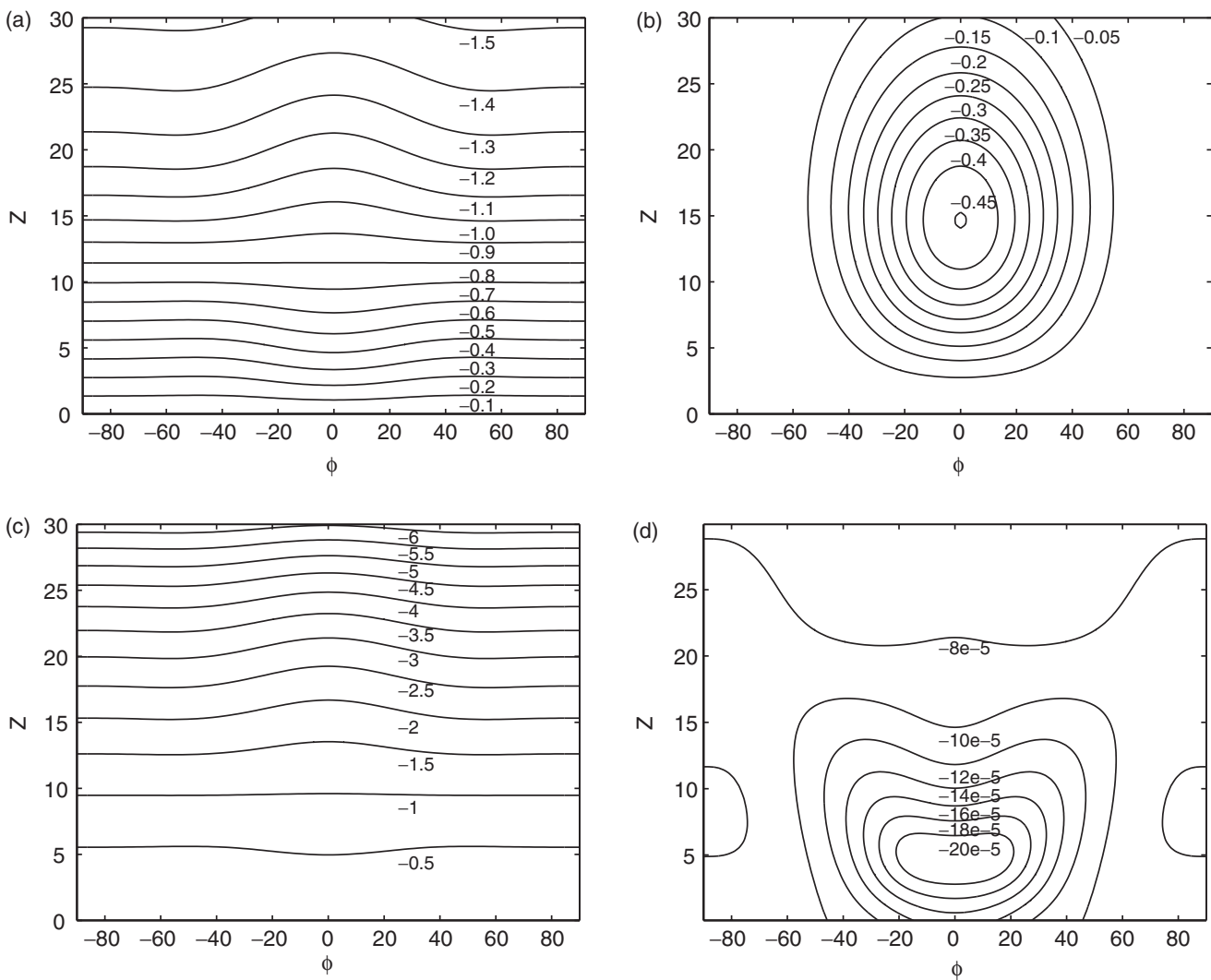

Figure 3. As Figure 1, but for basic-state (deep-atmosphere minus shallow-atmosphere) difference fields, and much reduced contour intervals: (a) $0.1 \mathrm{~K}$; (b) $0.05 \mathrm{~m} \mathrm{~s}^{-1}$; (c) $0.5 \mathrm{~K}$; and (d) $0.00002 \mathrm{~s}^{-1}$

consistency reasons, the gravitational acceleration in a shallowatmosphere model is constant (White et al., 2005; White and Wood, 2012), and this factor was only introduced in Eq. (13) to accommodate its vertical variation in a deep-atmosphere model. Thus (cf. Eq. (31) of SW11)

$$
T(\phi, r)=\left[\widetilde{\tau}_{1}(r)-\widetilde{\tau}_{2}(r)\left\{\cos ^{k} \phi-\left(\frac{k}{k+2}\right) \cos ^{k+2} \phi\right\}\right]^{-1},
$$

where $\tilde{\tau}_{1}(r)$ and $\widetilde{\tau}_{2}(r)$ are defined by Eqs (14) and (15), and $A, B$ and $C$ by Eqs (16) and (19).

SW11 show that the balancing flow is then given by their Eqs (32), (33) and (41). For simplicity, and consistency with the analogous choice made above for the deep-atmosphere case, the arbitrary integration function $F(a \cos \phi)$ in Eq. (32) of SW11 is set identically zero. The consequences of this are again that it leads to $u$ being identically zero at the surface $r=a$, and to also making $p=p_{0}$ there.

Note that there is no approximation involved here. Just as the assumed functional form (13) for $T(\phi, r)$ leads, by rigorous mathematical analysis, to exact solutions of the nonlinear deepatmosphere equations, so the assumed functional form (20) for $T(\phi, r)$ leads, by rigorous mathematical analysis, to exact solutions of the nonlinear shallow-atmosphere equations. Because the deep- and shallow-atmosphere equation sets are subtly different, so the appropriate assumed functional forms (13) and (20) for $T(\phi, r)$ to tractably obtain exact solutions are also subtly different. That said, the factor of $(a / r)^{2}$ in Eq. (13), which multiplies $\widetilde{\tau}_{1}(r)$ and $\widetilde{\tau}_{2}(r)$, equals unity to within one third of a percent for $z \equiv r-a<10 \mathrm{~km}$, i.e. throughout the depth of the troposphere. The assumed deep- and shallowatmosphere functional forms (13) and (20) for $T(\phi, r)$ are therefore quantitatively very close indeed to one another.

The proposed shallow-atmosphere configuration is summarised in Appendix B.

\section{Baroclinic wave triggering mechanism}

To trigger a baroclinic instability, JW06 add a perturbation to the zonal wind field $u$ which is of Gaussian form in the horizontal, 
Table 2. Parameters used in running the baroclinic instability test for each dynamical core at a grid spacing of approximately $1^{\circ}$.

\begin{tabular}{|c|c|}
\hline $\begin{array}{l}\text { Dynamical } \\
\text { core }\end{array}$ & Parameters \\
\hline MCore & $\begin{array}{l}\text { Deep/shallow, non-hydrostatic, no hyperdiffusion, third-order } \\
\text { Runge-Kutta in time, } \\
\Delta t=200 \mathrm{~s}\end{array}$ \\
\hline ENDGame & $\begin{array}{l}\text { Deep/shallow, non-hydrostatic, off-centring } \epsilon=0.002 \text {, no } \\
\text { polar filtering, } 2 \times 2 \text { iterations, } \\
\Delta t=3600 \mathrm{~s}\end{array}$ \\
\hline CAM-FV & $\begin{array}{l}\text { Shallow, hydrostatic, } 4 \text { th-order divergence damping option, } \\
\Delta t=180 \mathrm{~s}\end{array}$ \\
\hline CAM-SLD & $\begin{array}{l}\text { Shallow, hydrostatic, default off-centring } \epsilon=0.2 \text {, no diffusion, } \\
\text { energy fixer, } \\
\Delta t=1200 \mathrm{~s}\end{array}$ \\
\hline CAM-EUL & $\begin{array}{l}\text { Shallow, hydrostatic, } 4 \text { th-order hyperdiffusion dif } 4=0.5 \times \\
10^{15} \mathrm{~m}^{4} \mathrm{~s}^{-1} \text {, energy fixer, } \\
\Delta t=450 \mathrm{~s}\end{array}$ \\
\hline CAM-SE & $\begin{array}{l}\text { Shallow, hydrostatic, } 4 \text { th-order hyperdiffusion dif } 4=0.5 \times \\
10^{15} \mathrm{~m}^{4} \mathrm{~s}^{-1}, n_{p}=4, \\
\Delta t=360 \mathrm{~s}\end{array}$ \\
\hline
\end{tabular}

dif 4 denotes the coefficient of the $\nabla^{4}$ hyperdiffusion.

$n_{p}=4$ refers to the use of cubic polynomials in the spectral element method.

and uniform in the vertical. This is improved upon herein by instead perturbing both the zonal and meridional wind fields using a stream function

$$
\psi^{\prime}=-\frac{8 d_{0} V_{\mathrm{p}}}{3 \sqrt{3} \pi} \mathfrak{T}(z) \cos ^{4}\left(\frac{\pi d}{2 d_{0}}\right), \quad 0 \leq d \leq d_{0},
$$

where

$$
\mathfrak{T}(z) \equiv 1-3\left(\frac{z}{z_{\mathrm{t}}}\right)^{2}+2\left(\frac{z}{z_{\mathrm{t}}}\right)^{3}, \quad 0 \leq z \leq z_{\mathrm{t}},
$$

is a vertical taper function over depth $z_{\mathrm{t}}$ with zero derivative at $z=0$ and $z=z_{\mathrm{t}}$. Here

$$
d=a \cos ^{-1}\left\{\sin \phi_{\mathrm{c}} \sin \phi+\cos \phi_{\mathrm{c}} \cos \phi \cos \left(\lambda-\lambda_{\mathrm{c}}\right)\right\},
$$

is the Great Circle distance away from the geographic location $\left(\lambda_{c}, \phi_{c}\right), d_{0}$ defines the boundary of the horizontal domain over which the perturbation is applied, and $V_{\mathrm{p}}$ is the maximum value of the perturbed wind speed, which occurs at $z=0$ on $d=d_{0} / 3$. The perturbed zonal and meridional wind fields then follow from

$$
u^{\prime} \equiv-\frac{1}{a} \frac{\partial \psi^{\prime}}{\partial \phi}, \quad v^{\prime} \equiv \frac{1}{a \cos \phi} \frac{\partial \psi^{\prime}}{\partial \lambda},
$$
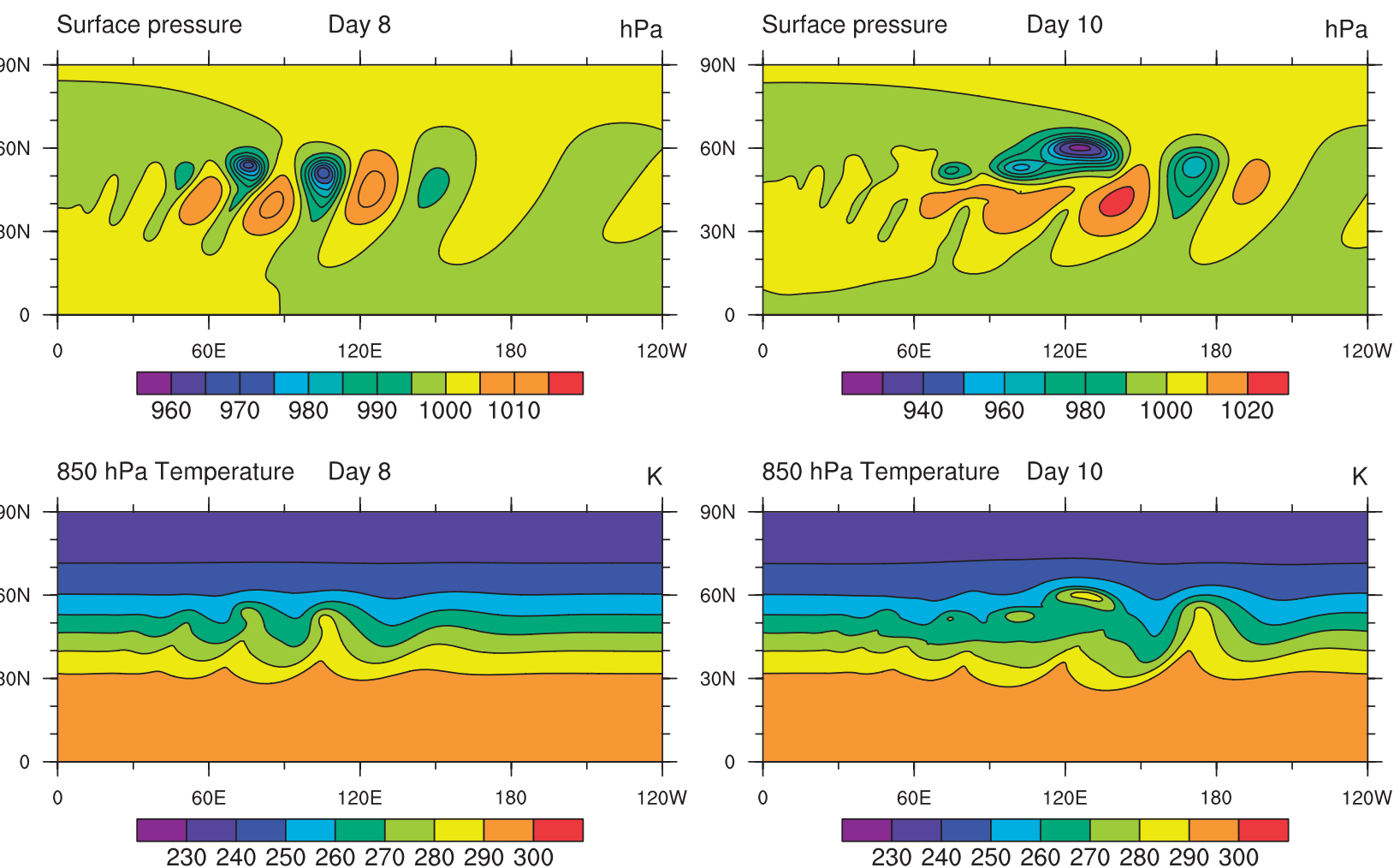

$$
\text { - } 90
$$
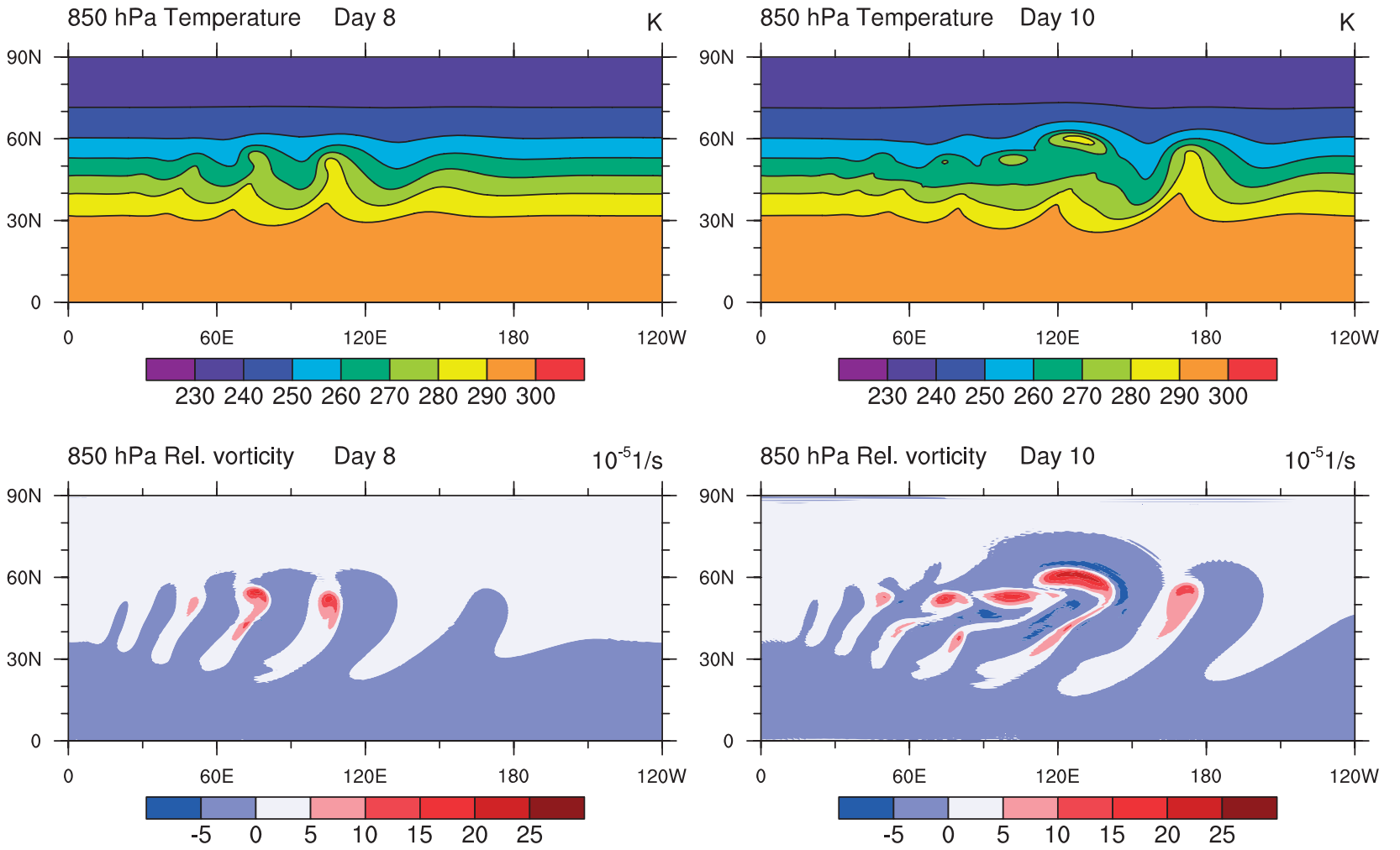

Figure 4. Results from MCore (deep-atmosphere) at days 8 (left panels) and 10 (right panels), showing (from top to bottom) surface pressure, 850 hPa temperature and $850 \mathrm{hPa}$ relative vorticity. The Northern Hemisphere between $0^{\circ}$ and $120^{\circ} \mathrm{W}$ longitude is shown, as other regions are visually indistinguishable from the initial state. 

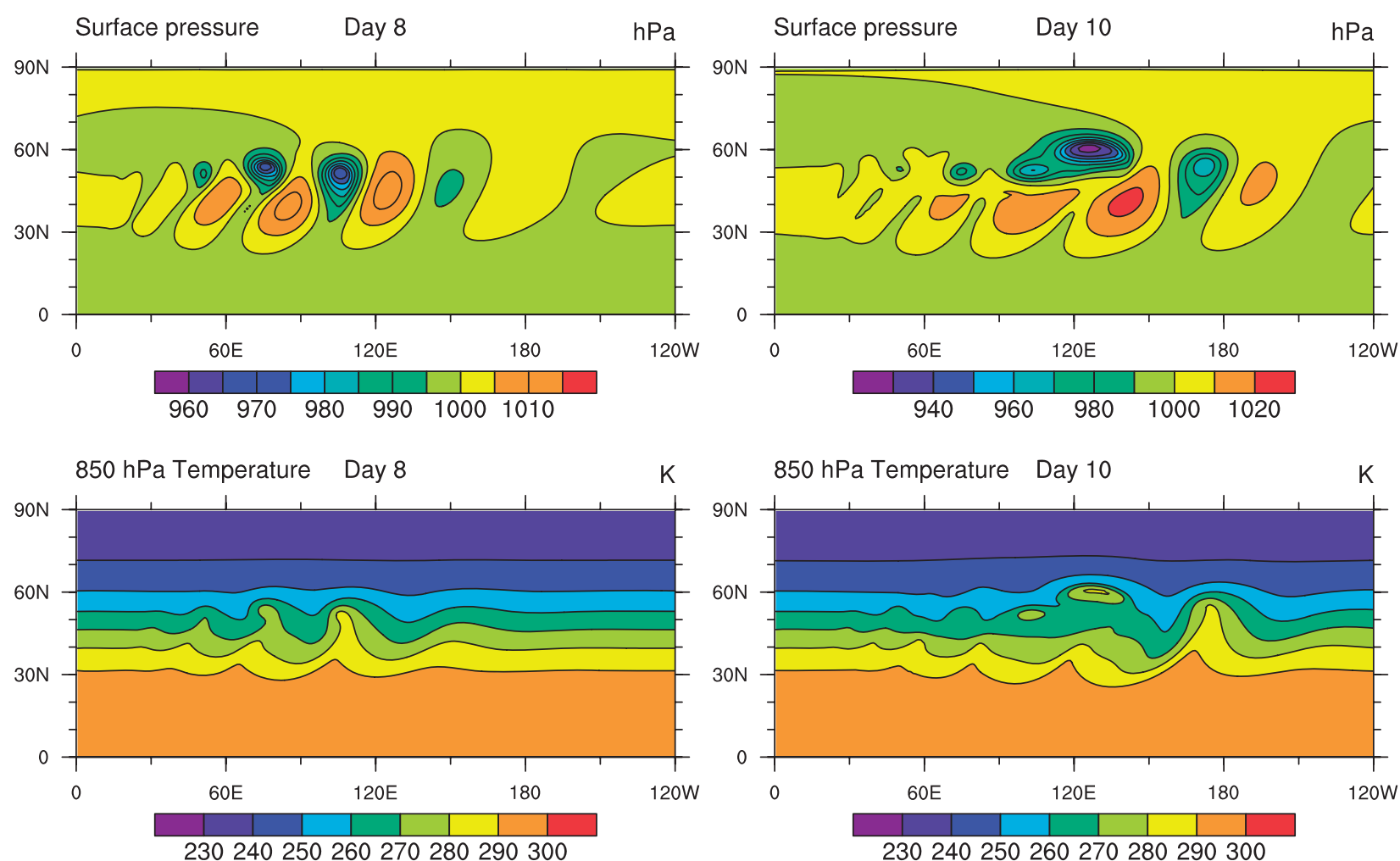

K
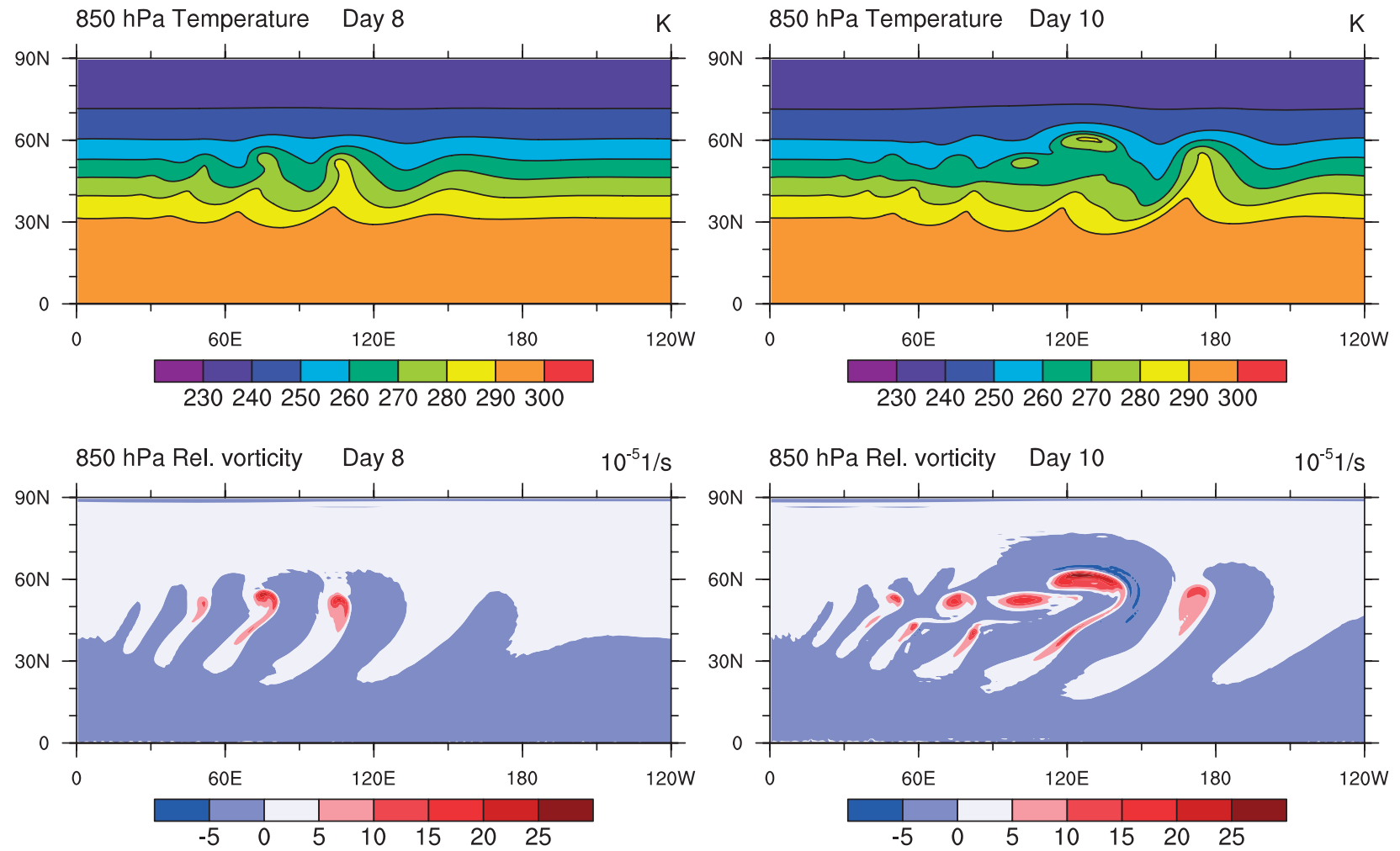

Figure 5. As Figure 4, but for ENDGame (deep-atmosphere).

in combination with Eq. (21), and lead to the explicit expressions

$$
\begin{aligned}
& u^{\prime}=- \frac{16 V_{\mathrm{p}}}{3 \sqrt{3}} \mathfrak{T}(z) \cos ^{3}\left(\frac{\pi d}{2 d_{0}}\right) \sin \left(\frac{\pi d}{2 d_{0}}\right) \\
& \times \frac{\left\{-\sin \phi_{\mathrm{c}} \cos \phi+\cos \phi_{\mathrm{c}} \sin \phi \cos \left(\lambda-\lambda_{\mathrm{c}}\right)\right\}}{\sin (d / a)}, \\
& v^{\prime}=\frac{16 V_{\mathrm{p}}}{3 \sqrt{3}} \mathfrak{T}(z) \cos ^{3}\left(\frac{\pi d}{2 d_{0}}\right) \sin \left(\frac{\pi d}{2 d_{0}}\right) \\
& \times \frac{\cos \phi_{\mathrm{c}} \sin \left(\lambda-\lambda_{\mathrm{c}}\right)}{\sin (d / a)} .
\end{aligned}
$$

When $d=0$ or $d=a \pi$, these expressions are singular and so require the additional specification $u^{\prime}=v^{\prime}=0$ at these points. (This is consistent with taking the appropriate limits in Eqs (25) and (26).)

The above specification improves on the JW06 perturbation in two ways. First, the use of a vertical taper function $\mathfrak{T}(z)$ avoids unnecessarily creating gravitational and acoustic oscillations in the upper atmosphere. Second, the use of a stream function selectively perturbs the vorticity (which is what drives the baroclinic instability mechanism) whilst significantly reducing the creation of undesirable gravitational and acoustic oscillations in the lower atmosphere due to significantly reduced divergence

at early time. Although the flow at the initial time is nondivergent, the divergence tendency is not; so, although there is still some gravitational and acoustic activity, it is much reduced.

\section{Configuring the basic state}

\subsection{Parameter settings}

Values used for the various parameters are displayed in Table 1. The six parameters $\left(R, \Omega, a, g, \gamma\right.$ and $\left.p_{0}\right)$, i.e. those that are common to both the present and to the JW06 studies, are set to the values used in JW06. The jet-configuration parameters $b, k, T_{0}^{\mathrm{P}}$ and $T_{0}^{\mathrm{E}}$ are set to the values used in SW12. The perturbed wind amplitude $V_{\mathrm{p}}$ and $d_{0}$ are chosen analogous to JW06. The parameter $z_{\mathrm{t}}$ is chosen to drive the perturbation to zero at $15 \mathrm{~km}$ altitude. The longitudinal centre of the perturbation $\lambda_{\mathrm{c}}$ is chosen to be slightly eastwards of $0^{\circ}$ to facilitate visualisation and constrain the initial development to $\lambda>0$. Finally, the latitudinal centre of the perturbation $\phi_{\mathrm{c}}$ is chosen to be slightly southwards of the centre of the jet to avoid bifurcating the baroclinic instability, as would occur if the perturbation were placed directly over the jet.

Regarding the value taken for $k$, JW06's midlatitude jets vary as $\sin ^{2}(2 \phi)$ along isobaric surfaces, so that the jet maxima are located at $\phi= \pm \pi / 4$ along any isobaric surface. For $u \ll 2 \Omega a$, 
(a) $50^{\circ} \mathrm{N}$ Day 8 Pressure perturbation $(\mathrm{hPa})$

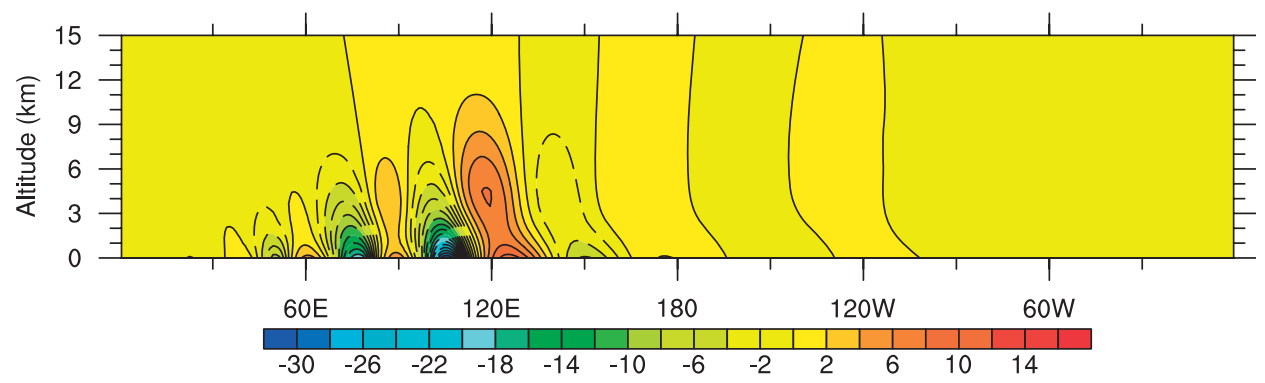

(b) $50^{\circ} \mathrm{N}$ Day 10 Pressure perturbation $(\mathrm{hPa})$

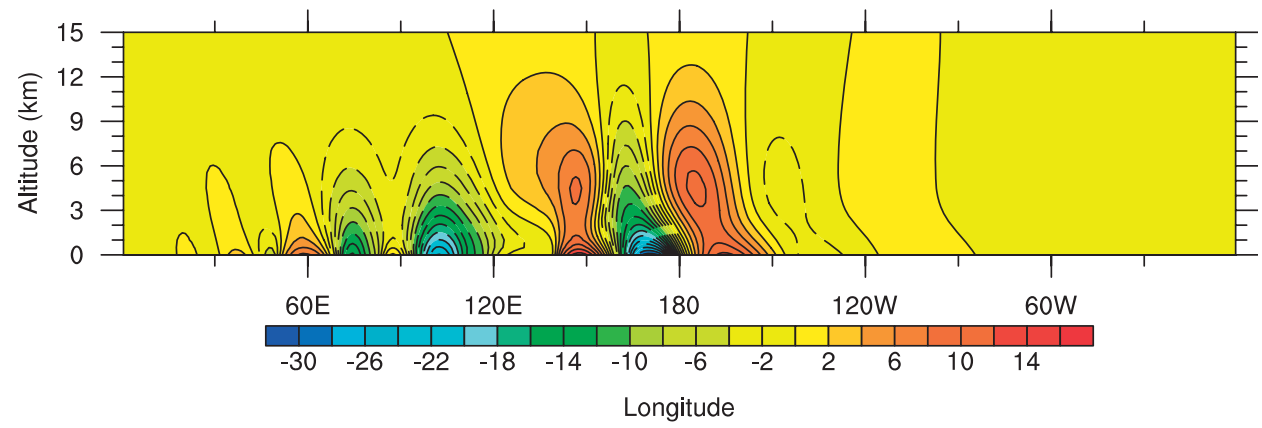

Figure 6. Cross-section through $50^{\circ} \mathrm{N}$ latitude of the perturbation of the pressure field from its initial state using MCore (deep-atmosphere) at (a) day 8 and (b) day 10. Dashed curves indicate negative contours. The zero line is in bold.

i.e. for small Rossby number, and for $r-a \ll a$, Eq. (A4) can be approximated as

$$
u(\phi, r) \approx \frac{g k}{2 \Omega a}\left(\cos ^{k-1} \phi-\cos ^{k+1} \phi\right) \int_{a}^{r} \tilde{\tau}_{2}\left(r^{\prime}\right) \mathrm{d} r^{\prime} T(\phi, r) .
$$

Setting $k=3$ then leads to $u \sim \sin ^{2}(2 \phi)$ along constant-height surfaces, provided that the modulating function $T(\phi, r)$ does not vary too strongly along these surfaces. This is in broad agreement with JW06, under the assumption that isobaric and constant-height surfaces do not differ from one another too significantly. Thus $k \equiv 3$ is an appropriate choice for configuring a baroclinic-wave test problem analogous to the JW06 one.

\subsection{Characteristics of the basic state}

The initial temperature, zonal wind, potential temperature and Brunt-Väisälä frequency fields for a deep atmosphere, corresponding to the parameter values of Table 1, are displayed in Figure 1, and some vertical profiles of the temperature, zonal-wind and pressure fields in Figure 2.

The depicted fields approximate the primary dynamic characteristics of Earth's atmosphere, with a single westerly jet of magnitude $\sim 28 \mathrm{~m} \mathrm{~s}^{-1}$ in each hemisphere and an equatorial belt with weak static stability. As mentioned previously, unlike the baroclinic instability test of JW06, this formulation does not include a vertical temperature inversion which might be associated with an idealised tropopause.

Because $r / a$ varies so little over the lowest $30 \mathrm{~km}$ of the atmosphere, the corresponding fields and profiles for a shallow atmosphere are almost identical, as evidenced by the difference fields of Figure 3. It is seen that the impact of $r / a$ being replaced by unity leads to differences that are of very large horizontal and vertical scale, and of very small amplitude.

\subsection{Numerical determination of $r$ from given values of pressure and latitude}

For a model with a vertical coordinate based on spherical radius/ geometric height, it is straightforward to evaluate the

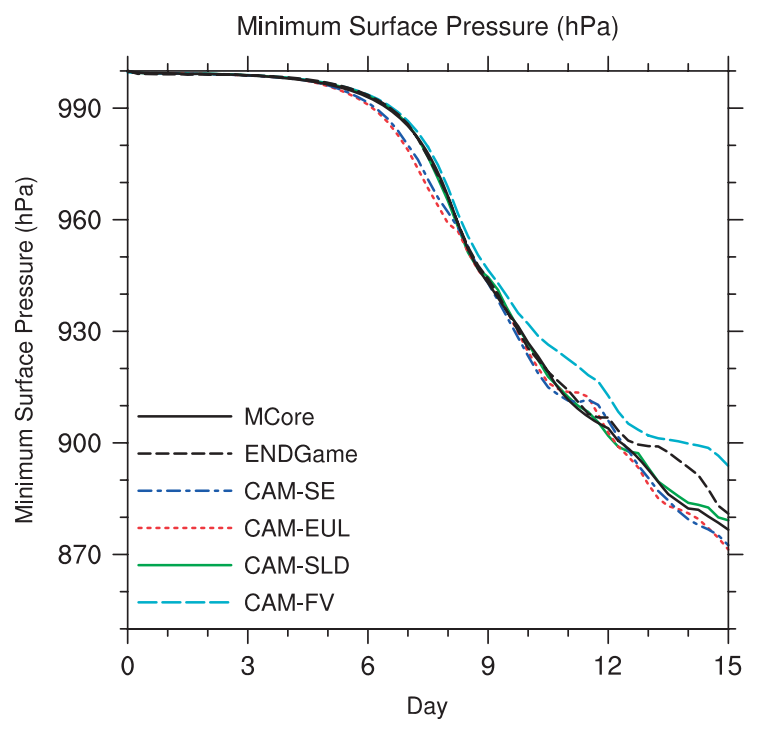

Figure 7. Minimum surface pressure for the suite of dynamical cores given in Table 2 running the shallow-atmosphere baroclinic instability test for a 15 day simulation.

dependent variables on a coordinate surface. This is because the deep- and shallow-atmosphere basic states are most naturally expressed in terms of the spherical polar coordinate $r$. For a model based on pressure, a way is needed of accurately determining the value of $r$ which corresponds to given values of pressure $p$ and latitude $\phi$. Newton iteration satisfies this requirement and, as applied to the present work, the associated procedure is given in Appendix $\mathrm{C}$ for both deep and shallow atmospheres.

\subsection{Initialisation routine}

To aid in the implementation and intercomparison of results using different atmospheric models, a Fortran initialisation routine has been provided as an online adjunct to this article. The initialisation routine umjsbcinst. $\mathrm{f} 90$ includes comments to explain how it can be used for models that employ either a height-based or a pressure-based vertical coordinate. 
MCore

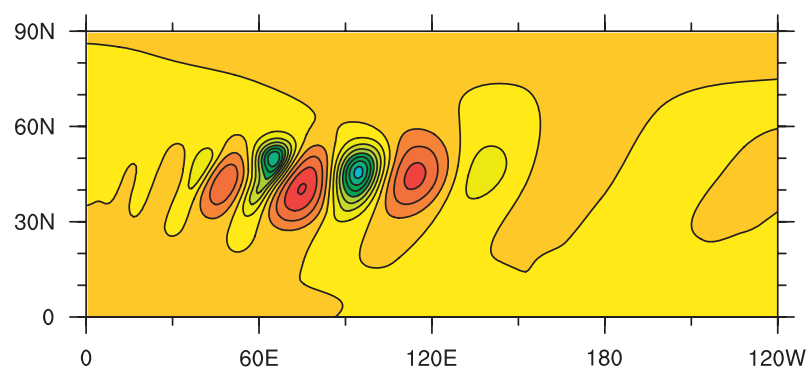

CAM-FV

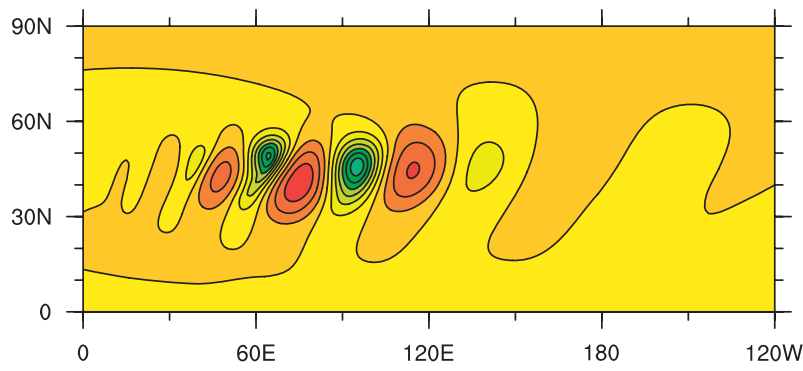

CAM-EUL

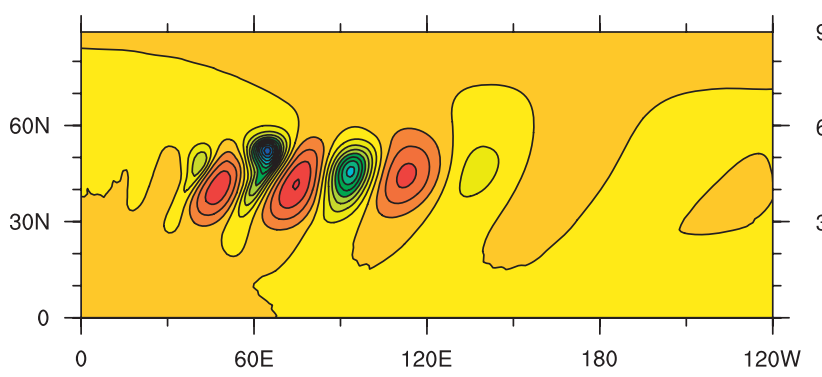

ENDGame

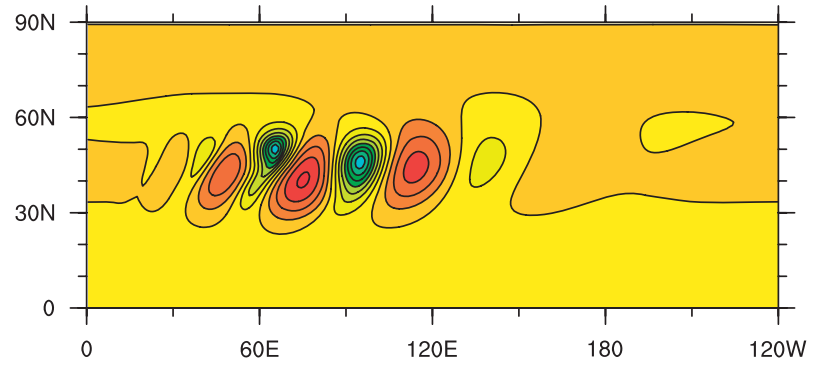

CAM-SLD

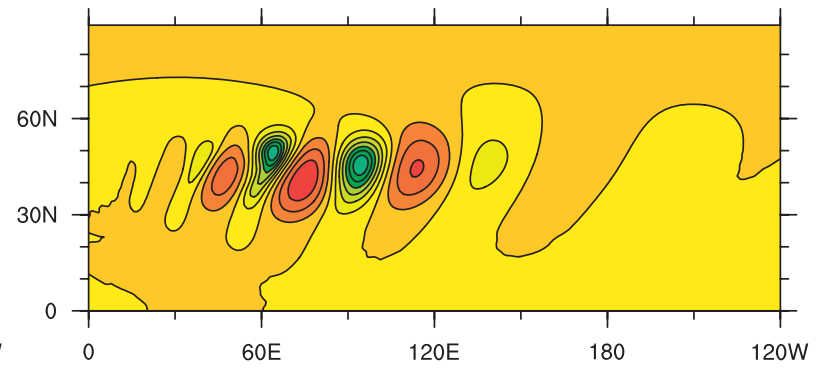

CAM-SE

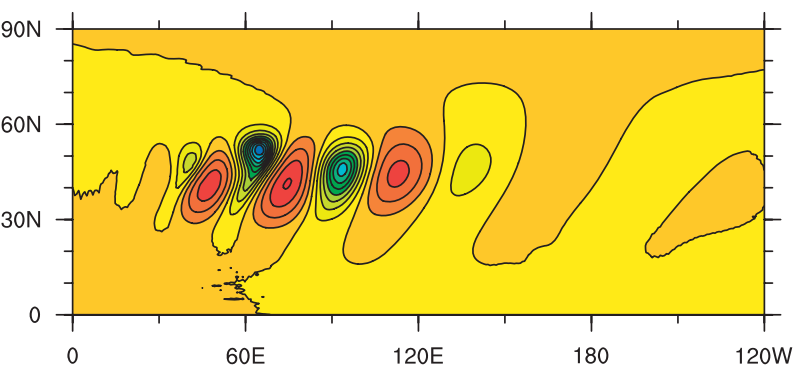

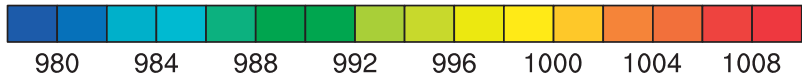

Figure 8. Northern Hemisphere surface pressure fields ( $\mathrm{hPa}$ ) at day 7 for the suite of dynamical cores given in Table 2. MCore and ENDGame are run using deep-atmosphere configurations.

\section{Illustrative results}

\subsection{Evolution of the baroclinic instability}

Holton (1992), for example, gives a discussion of the theoretical properties governing evolution of the baroclinic instability in the context of quasi-geostrophic theory.

The initial state described in sections 3 to 6 was implemented in the MCore (Ullrich and Jablonowski, 2012a) and ENDGame (Wood et al., 2013) non-hydrostatic atmospheric dynamical cores and run for a period of 15 days. Specific parameter values used for each simulation are given in Table 2. MCore was run with a $90 \times 90$ array of elements on each panel of a cubed sphere (corresponding to $1^{\circ}$ resolution along the Equator) and a time step of $\Delta t=200 \mathrm{~s}$. ENDGame was run with a global resolution of $1^{\circ}$ on a regular lat-lon grid with a time step of $\Delta t=3600 \mathrm{~s}$. Both models were configured to have 30 vertical levels and a model top at $z_{\text {top }}=30 \mathrm{~km}$. A non-uniform distribution of vertical levels was chosen to enhance resolution near the surface, where the vertical shear is strong. Specifically, the height of the $n$th model interface $z_{n}$ (with $n=0,1, \ldots, 30$ ) is given by

$$
z_{n}=z_{\text {top }} \frac{\left\{\mu(n / 30)^{2}+1\right\}^{1 / 2}-1}{(\mu+1)^{1 / 2}-1},
$$

where $\mu=15$ is the flattening parameter. Model levels are spaced half way between model interfaces. Results after 8 and 10 simulation days using a full deep-atmosphere configuration are shown in Figure 4 for MCore and Figure 5 for ENDGame. Shallow-atmosphere simulations were also run, but the results were visually indistinguishable from the deep-atmosphere ones and so are not shown.

The two model integrations agree very well until day 8 , when wave-breaking occurs, as evidenced by overturning in the temperature field. The linear theory governing the evolution of a baroclinic instability predicts exponential growth of the most unstable mode and the results are consistent with this until approximately day 8 . After day 8 , nonlinear effects become important, and consequently the behaviour of models for this test can be expected to quickly diverge thereafter.

A cross-section through the perturbed pressure field of the baroclinic instability at $50^{\circ} \mathrm{N}$ is shown in Figure 6 for the MCore simulation. The perturbation shows the characteristic westward shift with height observed for baroclinic instability in a channel. This westward shift with altitude is a characteristic signature of the most unstable mode of a baroclinic instability.

\subsection{Surface pressure evolution}

The initialisation procedure described above was also implemented in the Community Atmosphere Model framework (CAM; Neale et al., 2010) using the parameters specified in Table 2. The CAM version 5 dynamical core suite includes the spectraltransform semi-Lagrangian (SLD), the spectral-transform Eulerian (EUL), the Finite-Volume (FV) and the Spectral Element (SE) dynamical cores. SLD and EUL were run with the triangular 
Shallow Atmosphere $(X=20)$
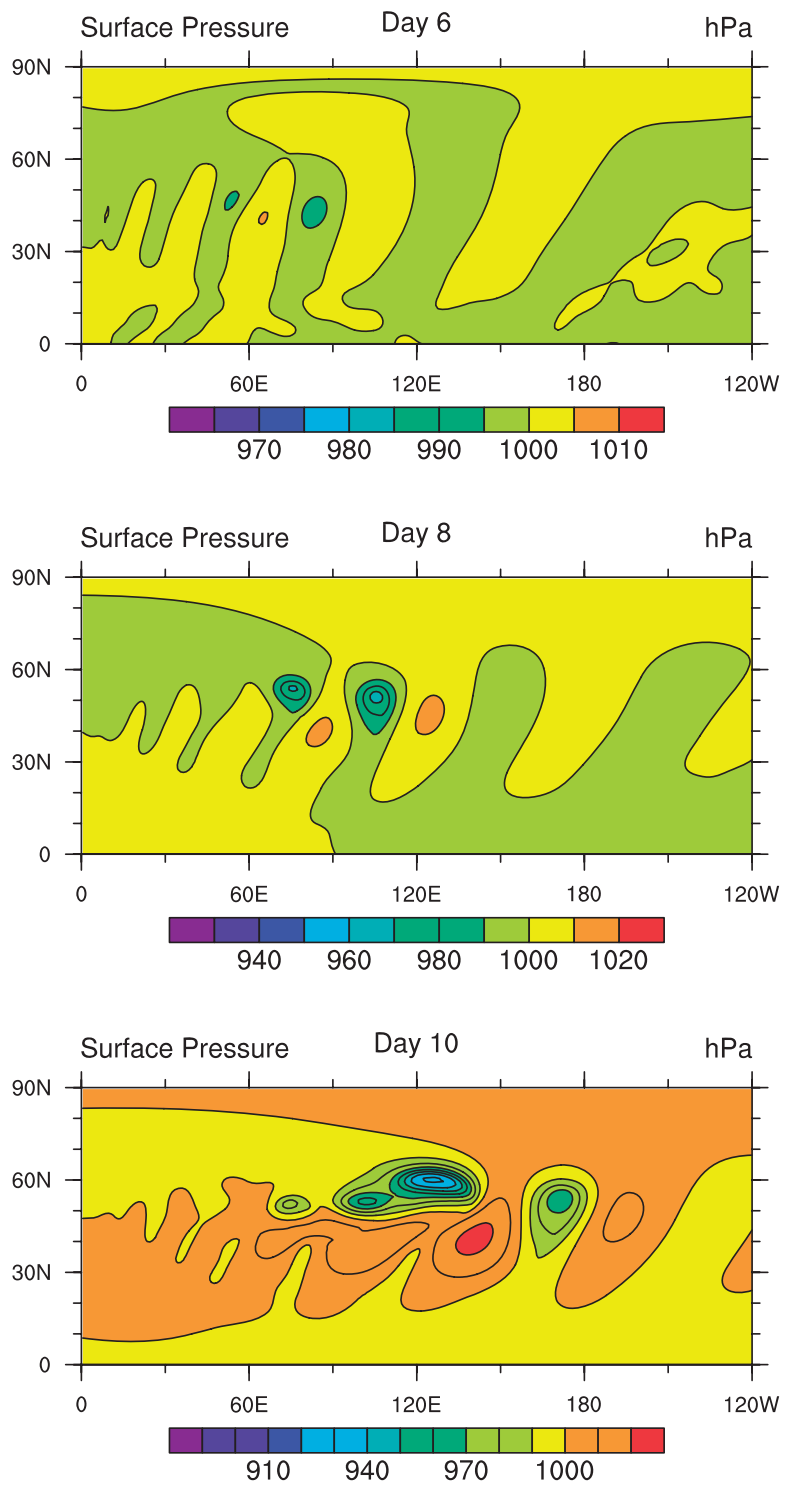

Deep Atmosphere $(X=20)$
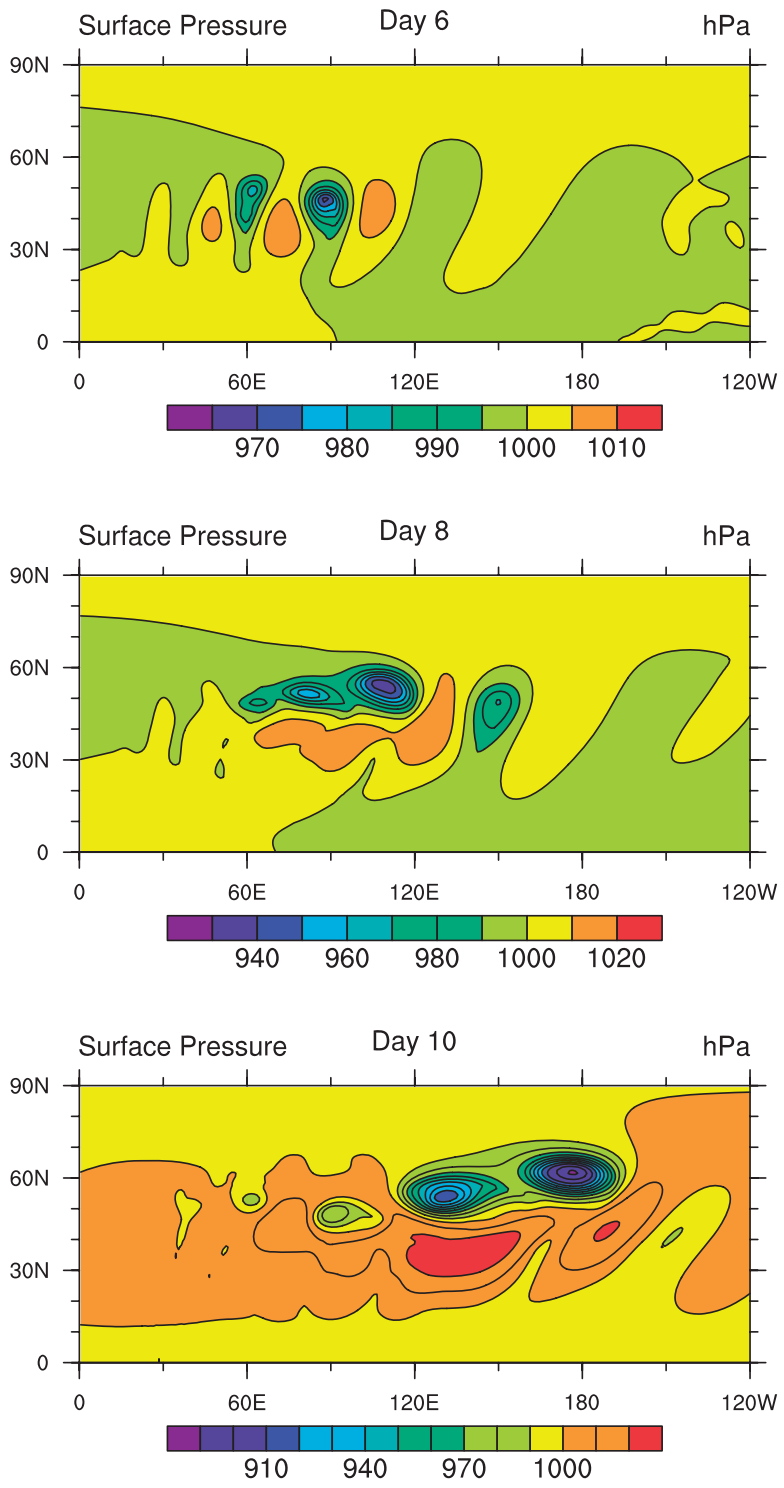

Figure 9. Surface pressure for the shallow-atmosphere and deep-atmosphere formulations simulated using MCore and the small-Earth approach with a scaling factor of $X=20$ and time step $\Delta t=10 \mathrm{~s}$. Results are plotted (from top to bottom) at (scaled) days 6, 8 and 10 , corresponding to 25920,34560 and $43200 \mathrm{~s}$ of unscaled simulation time.

truncation T106 which corresponds to a Gaussian grid spacing of about $1.125^{\circ}$. FV and SE were run with $1^{\circ} \times 1^{\circ}$ grid spacings which are approximate values for the cubed-sphere grid of the SE model. All CAM models used 30 vertical levels with a model top around $2 \mathrm{hPa}$. The distribution of the hybrid pressurebased vertical levels follows the CAM version 5 default, with enhanced resolution near the surface (Reed and Jablonowski, 2012, their Appendix B). A plot of the minimum surface pressure over time from this suite of dynamical cores is given in Figure 7.

Three of the models follow a similar evolution up to day 8, including CAM-SLD, ENDGame and MCore. However, CAMFV displays slightly weaker growth relative to the other models, whereas CAM-EUL and CAM-SE show stronger development of the wave, especially between days 5 and 8 . The overall qualitative features of the wave are identical in all six models as shown in Figure 8 for the surface pressure at day 7 . However, these results suggest some sensitivity of the developing wave to the dynamic character of each model. Notably, for this suite of models these results do not seem to be very sensitive to further refinement in horizontal or vertical resolution. Many of the models were tested at higher resolution (roughly $0.5^{\circ}$ equatorial resolution) with essentially no change in the day 6 observed minimum pressures, and with only a small intensification $(\sim 2 \mathrm{hPa})$ in the day 8 observed minimum pressures of MCore, CAM-FV and CAM-SLD.

\subsection{Small-Earth experiments}

As noted earlier, the shallow-atmosphere and deep-atmosphere formulations of the baroclinic instability test produce visually indistinguishable results over the integration period for the given choice of parameters. The small-Earth framework of Wedi and Smolarkiewicz (2009) is helpful in this situation to exaggerate differences between shallow- and deep-atmosphere formulations. It thereby allows a user to specifically test the deep-atmosphere aspects of the formulation of a deepatmosphere model.

Following this approach, the MCore model was run in both shallow-atmosphere and deep-atmosphere modes with a new small-Earth radius of $a^{*} \equiv a / X$ and increased rotation rate of $\Omega^{*} \equiv \Omega X$, where $X=20$ is the small-Earth scaling factor. The baroclinic instability was evolved until time $43200 \mathrm{~s}$, which corresponds to 10 days (complete rotations) on the small Earth, with a time step of $\Delta t=10 \mathrm{~s}$. Significant differences in the surface pressure of the baroclinic instability can be observed, even very early in the simulation, as shown in Figure 9. In particular, the shallow-atmosphere results closely match those of Figure 4; this is 
not unexpected since $X=20$ is insufficient to drive the simulation to scales where non-hydrostatic effects are relevant for largescale dynamics. However, the results from the deep-atmosphere integration show a significantly more rapid intensification of the wave, which has already driven the minimum pressure below $900 \mathrm{hPa}$ by day 10 .

This experiment was repeated with the ENDGame dynamical core, run with a time step of $\Delta t=180 \mathrm{~s}$, producing similar results (not shown) to those of Figure 9.

\section{Summary and conclusion}

A proposed formulation of an idealised baroclinic instability test has been developed herein for deep- and shallow-atmosphere models. Equivalence between the deep- and shallow-atmosphere formulations holds in the limit $r / a \rightarrow 1$. The initial conditions feature a constant surface pressure, and so are well-suited to both height-based and pressure-based terrain-following coordinate models. An improved wave-triggering mechanism has also been introduced, featuring a vertical tapering of the perturbation to eliminate undesirable oscillations in the upper atmosphere. Furthermore, the perturbation is constructed to selectively target the vorticity field and thereby reduce the initial contamination of the solution by rapidly propagating gravity waves.

This proposed test case has been run in the non-hydrostatic deep-atmosphere MCore and ENDGame dynamical cores, as well as the hydrostatic shallow-atmosphere dynamical cores from the CAM framework. Furthermore, output after 8 and 10 simulated days has been provided to facilitate the future development of this proposal into a fully-fledged test case. Small-planet tests have also been run with the purpose of exaggerating differences between the deep- and shallowatmosphere formulations.

The test case formulated here is effective at capturing the essential physical features of a baroclinic wave in an idealised setting, and presents a formulation which is a natural improvement to prior approaches to modelling this phenomenon. This proposed test case is the first in a proposed series of standard idealised test cases which bridge the gap between deep- and shallow-atmosphere models, and hence is of importance for model development and intercomparison efforts. Further, it opens up a path for defining, in a similarly unified manner, an analogous baroclinic-wave test problem for deep- and shallow-atmosphere models in $\beta$ - and $\beta-\gamma$-plane geometries as reviewed by Staniforth (2012).

\section{Acknowledgements}

The authors gratefully acknowledge helpful discussions during the course of this work with Markus Gross, Sean Milton, Chris Smith, John Thuburn, Simon Vosper, Andy White, Nigel Wood and Colin Zarzycki. Christiane Jablonowski was supported by the Office of Science, US Department of Energy, Award No. DE-SC0006684.

\section{Appendix A. The deep-atmosphere basic state}

Integrating Eqs (14) and (15) gives

$$
\begin{aligned}
\int_{a}^{r} \tilde{\tau}_{1}\left(r^{\prime}\right) \mathrm{d} r^{\prime}= & A\left[\exp \left\{\frac{\Gamma}{T_{0}}(r-a)\right\}-1\right] \\
& +B(r-a) \exp \left\{-\left(\frac{r-a}{b H}\right)^{2}\right\}, \\
\int_{a}^{r} \tilde{\tau}_{2}\left(r^{\prime}\right) \mathrm{d} r^{\prime}= & C(r-a) \exp \left\{-\left(\frac{r-a}{b H}\right)^{2}\right\} .
\end{aligned}
$$

The basic-state temperature field $T(\phi, r)$ is obtained from Eq. (13), i.e. from

$$
\begin{aligned}
& T(\phi, r)= \\
& \left(\frac{a}{r}\right)^{2}\left[\tilde{\tau}_{1}(r)-\tilde{\tau}_{2}(r)\left\{\left(\frac{r}{a} \cos \phi\right)^{k}-\left(\frac{k}{k+2}\right)\left(\frac{r}{a} \cos \phi\right)^{k+2}\right\}\right]^{-1},
\end{aligned}
$$

where $\tilde{\tau}_{1}(r)$ and $\tilde{\tau}_{2}(r)$ are given by Eqs $(14)-(15)$.

Setting $F(r \cos \phi)$ identically zero in Eq. (12) of SW 11, the wind proxy $U(\phi, r)$ is given by

$$
\begin{aligned}
U(\phi, r) \equiv & 2 \Omega u+\frac{u^{2}}{r \cos \phi} \\
= & \frac{g}{a} k T(\phi, r) \int_{a}^{r} \tilde{\tau}_{2}\left(r^{\prime}\right) \mathrm{d} r^{\prime} \\
& \times\left\{\left(\frac{r \cos \phi}{a}\right)^{k-1}-\left(\frac{r \cos \phi}{a}\right)^{k+1}\right\},
\end{aligned}
$$

where $\int_{a}^{r} \widetilde{\tau}_{2}\left(r^{\prime}\right) \mathrm{d} r^{\prime}$ is given by Eq. (A2).

The wind field $u(\phi, r)$ is then obtained from Eq. (A4) (cf. Eq. (13) of SW11) by solving it as a quadratic, so that

$$
u(\phi, r)=-\Omega r \cos \phi+\sqrt{\Omega^{2} r^{2} \cos ^{2} \phi+r \cos \phi U(\phi, r)} .
$$

Finally, the pressure field is obtained by setting $Q(r \cos \phi)$ identically zero in Eq.(24) of SW11, so that

$$
\begin{aligned}
& p(\phi, r)=p_{0} \exp \left[-\frac{g}{R} \int_{a}^{r} \tilde{\tau}_{1}\left(r^{\prime}\right) \mathrm{d} r^{\prime}\right. \\
& \left.+\frac{g}{R} \int_{a}^{r} \tilde{\tau}_{2}\left(r^{\prime}\right) \mathrm{d} r^{\prime}\left\{\left(\frac{r \cos \phi}{a}\right)^{k}-\frac{k}{(k+2)}\left(\frac{r \cos \phi}{a}\right)^{k+2}\right\}\right],
\end{aligned}
$$

where $\int_{a}^{r} \widetilde{\tau}_{1}\left(r^{\prime}\right) \mathrm{d} r^{\prime}$ and $\int_{a}^{r} \widetilde{\tau}_{2}\left(r^{\prime}\right) \mathrm{d} r^{\prime}$ are given by Eqs (A1)-(A2).

Note that Eqs (A3)-(A6) correspond to Eqs (47), (41), (42) and (37), respectively, of SW12.

\section{Appendix B. The shallow-atmosphere basic state}

The basic-state temperature field $T(\phi, r)$ is obtained from Eq. (20), i.e. from

$$
T(\phi, r)=\left[\tilde{\tau}_{1}(r)-\tilde{\tau}_{2}(r)\left\{\cos ^{k} \phi-\left(\frac{k}{k+2}\right) \cos ^{k+2} \phi\right\}\right]^{-1},
$$

where $\tilde{\tau}_{1}(r)$ and $\tilde{\tau}_{2}(r)$ are given by Eqs (14) and (15).

Setting $F(a \cos \phi)$ identically zero in Eq. (32) of SW11, the wind proxy $U(\phi, r)$ is given by

$$
U(\phi, r)=\frac{g}{a} k \int_{a}^{r} \tilde{\tau}_{2}\left(r^{\prime}\right) \mathrm{d} r^{\prime}\left(\cos ^{k-1} \phi-\cos ^{k+1} \phi\right) T(\phi, r),
$$

where $\int_{a}^{r} \tilde{\tau}_{2}\left(r^{\prime}\right) \mathrm{d} r^{\prime}$ is given by Eq. (A2).

The wind field $u(\phi, r)$ is then obtained from Eq. (33) of SW11, so that

$$
u(\phi, r)=-\Omega a \cos \phi+\sqrt{\Omega^{2} a^{2} \cos ^{2} \phi+a \cos \phi U(\phi, r)} .
$$

Finally, the pressure field is obtained by setting $F(a \cos \phi)$ identically zero in Eq. (41) of SW11, so that

$$
\begin{aligned}
p(\phi, r)= & p_{0} \exp \left[-\frac{g}{R} \int_{a}^{r} \tilde{\tau}_{1}\left(r^{\prime}\right) \mathrm{d} r^{\prime}\right. \\
& \left.+\frac{g}{R} \int_{a}^{r} \tilde{\tau}_{2}\left(r^{\prime}\right) \mathrm{d} r^{\prime}\left\{\cos ^{k} \phi-\left(\frac{k}{k+2}\right) \cos ^{k+2} \phi\right\}\right],
\end{aligned}
$$


where $\int_{a}^{r} \widetilde{\tau}_{1}\left(r^{\prime}\right) \mathrm{d} r^{\prime}$ and $\int_{a}^{r} \widetilde{\tau}_{2}\left(r^{\prime}\right) \mathrm{d} r^{\prime}$ are given by Eqs (14) and as

(15).

\section{Appendix C. Numerical determination of $r$ from given values of $p$ and $\phi$}

\section{C1. Deep atmosphere}

Assume that specific values are prescribed for pressure $p$, and latitude $\phi$, and that one wishes to obtain the corresponding value of $r$ from Eq. (A6). This can be accomplished by defining

$$
\begin{aligned}
F(\phi, r) & \equiv \ln \left(\frac{p}{p_{0}}\right)+\frac{g}{R} \int_{a}^{r} \tilde{\tau}_{1}\left(r^{\prime}\right) \mathrm{d} r^{\prime} \\
& -\frac{g}{R} \int_{a}^{r} \tilde{\tau}_{2}\left(r^{\prime}\right) \mathrm{d} r^{\prime}\left\{\left(\frac{r \cos \phi}{a}\right)^{k}-\frac{k}{(k+2)}\left(\frac{r \cos \phi}{a}\right)^{k+2}\right\},
\end{aligned}
$$

(which corresponds to rewriting Eq. (A6) in logarithmic form) and then using Newton iteration to obtain

$$
r^{(n+1)}=r^{(n)}-\frac{F\left(\phi, r^{(n)}\right)}{\left.[\partial F(\phi, r) / \partial r]\right|_{r=r^{(n)}}} .
$$

Here $n$ denotes the iteration count and the initial estimate is chosen to be $r^{(0)}=a+10 \mathrm{~km}$. In Eq. (C2), $F\left(\phi, r^{(n)}\right)$ is straightforwardly obtained from Eq. (C1) by explicit evaluation at $r=r^{(n)}$. Typically 5-10 iterations are needed for convergence to machine precision.

Differentiating Eq. (C1) with respect to $r$ gives

$$
\begin{aligned}
& \frac{\partial F(\phi, r)}{\partial r}=\frac{g}{R} \tilde{\tau}_{1}(r)-\frac{g}{R} \tilde{\tau}_{2}(r) \\
& \times\left\{\left(\frac{r \cos \phi}{a}\right)^{k}-\frac{k}{(k+2)}\left(\frac{r \cos \phi}{a}\right)^{k+2}\right\} \\
& -\frac{g}{R} k \frac{\cos \phi}{a} \int_{a}^{r} \tilde{\tau}_{2}\left(r^{\prime}\right) \mathrm{d} r^{\prime}\left\{\left(\frac{r \cos \phi}{a}\right)^{k-1}-\left(\frac{r \cos \phi}{a}\right)^{k+1}\right\} .
\end{aligned}
$$

Using Eqs (14),(15) and (A2) in this equation yields the explicit formula

$$
\begin{aligned}
\frac{\partial F(\phi, r)}{\partial r}= & A \frac{g}{R} \frac{\Gamma}{T_{0}} \exp \left\{\frac{\Gamma}{T_{0}}(r-a)\right\} \\
& -C \frac{g}{R} k \frac{\cos \phi}{a}(r-a) \exp \left\{-\left(\frac{r-a}{b H}\right)^{2}\right\} \\
& \times\left\{\left(\frac{r \cos \phi}{a}\right)^{k-1}-\left(\frac{r \cos \phi}{a}\right)^{k+1}\right\} \\
& +\frac{g}{R}\left\{1-2\left(\frac{r-a}{b H}\right)^{2}\right\} \exp \left\{-\left(\frac{r-a}{b H}\right)^{2}\right\} \\
& \times\left[B-C\left\{\left(\frac{r \cos \phi}{a}\right)^{k}-\frac{k}{(k+2)}\left(\frac{r \cos \phi}{a}\right)^{k+2}\right\},\right.
\end{aligned}
$$

which is then evaluated at $r=r^{(n)}$.

Finally, Eq. (C2) is applied iteratively to obtain the value of $r$ that corresponds to given values of $p$ and $\phi$.

\section{C2. Shallow atmosphere}

The analogous procedure for the shallow atmosphere is identical to that for the deep atmosphere except that $F(\phi, r)$ is redefined

$$
\begin{aligned}
F(\phi, r) \equiv & \ln \left(\frac{p}{p_{0}}\right)+\frac{g}{R} \int_{a}^{r} \tilde{\tau}_{1}\left(r^{\prime}\right) \mathrm{d} r^{\prime} \\
& -\frac{g}{R} \int_{a}^{r} \tilde{\tau}_{2}\left(r^{\prime}\right) \mathrm{d} r^{\prime}\left\{\cos ^{k} \phi-\left(\frac{k}{k+2}\right) \cos ^{k+2} \phi\right\} .
\end{aligned}
$$

Differentiating Eq. (C5) with respect to $r$, and using Eqs (14) and (15), then yields the explicit formula

$$
\begin{aligned}
\frac{\partial F(\phi, r)}{\partial r}= & A \frac{g}{R} \frac{\Gamma}{T_{0}} \exp \left\{\frac{\Gamma}{T_{0}}(r-a)\right\} \\
& +\frac{g}{R}\left\{1-2\left(\frac{r-a}{b H}\right)^{2}\right\} \exp \left\{-\left(\frac{r-a}{b H}\right)^{2}\right\} \\
& \times\left[B-C\left\{\cos ^{k} \phi-\left(\frac{k}{k+2}\right) \cos ^{k+2} \phi\right\}\right],
\end{aligned}
$$

instead of Eq. (C4). Equation (C2) is then applied iteratively to obtain the value of $r$ that corresponds to given values of $p$ and $\phi$.

\section{References}

Cullen MJP. 1993. The unified forecast/climate model. Meteorol. Mag. 122: 81-94.

Davies T, Cullen MJP, Malcolm A, Mawson M, Staniforth A, White AA, Wood N. 2005. A new dynamical core for the Met Office's global and regional modelling of the atmosphere. Q. J. R. Meteorol. Soc. 131: $1759-1782$.

Holton JR. 1992. An Introduction to Dynamic Meteorology (3rd edn). Academic Press: San Diego, CA.

Hoskins BJ, Simmons AJ. 1975. A multi-layer spectral model and the semiimplicit method. Q. J. R. Meteorol. Soc. 101: 637-655.

Jablonowski C, Williamson DL. 2006a. A baroclinic instability test case for atmospheric model dynamical cores. Q. J. R. Meteorol. Soc. 132: 2943-2975.

Jablonowski C, Williamson DL. 2006b. 'A baroclinic wave test case for dynamical cores of general circulation models: model intercomparisons', Technical report TN-469+STR. NCAR: Boulder, CO http://www.library.ucar.edu/uhtbin/hyperion-image/DR000790 (accessed 15 October 2013)

Lauritzen PH, Jablonowski C, Taylor MA, Nair RD. 2010. Rotated versions of the Jablonowski steady-state and baroclinic wave test cases: a dynamical core intercomparison. J. Adv. Model. Earth Syst. 2: 15, doi: 10.3894/JAMES.2010.2.15.

Läuter M, Handorf D, Dethloff K. 2005. Unsteady analytical solutions of the spherical shallow water equations. J. Comput. Phys. 210: 535-553.

Neale RB, Chen CC, Gettelman A, Lauritzen PH, Park S, Williamson DL, Conley AJ, Garcia R, Kinnison D, Lamarque JF, Marsh D, Mills M, Smith AK, Tilmes S, Vitt F, Cameron-Smith P, Collins WD, Iacono MJ, Easter RC, Liu X, Ghan SJ, Rasch PJ, Taylor MA. 2010. 'Description of the NCAR Community Atmosphere Model (CAM 5.0)', Technical Note NCAR/TN-486+STR. NCAR: Boulder, CO. http://www.cesm.ucar.edu/models/cesm1.0/cam (accessed 15 October 2013).

Reed KA, Jablonowski C. 2012. Idealized tropical cyclone simulations of intermediate complexity: A test case for AGCMs. J. Adv. Model. Earth Syst. 4: M04001, doi: 10.1029/2011MS000099.

Satoh M, Matsuno T, Tomita H, Miura H, Nasuno T, Iga S. 2008 Non-hydrostatic icosahedral atmospheric model (NICAM) for global cloud-resolving simulations. J. Comput. Phys. 227: 3486-3514, doi: 10.1016/j.jcp.2007.02.006

Simmons AJ, Hoskins BJ. 1975. A comparison of spectral and finite-difference simulations of a growing baroclinic wave. Q. J. R. Meteorol. Soc. 101: 551-565.

Staniforth A. 2012. Exact stationary axisymmetric solutions of the Euler equations on beta-gamma planes. Atmos. Sci. Lett. 13: 79-87.

Staniforth A, White AA. 2007. Some exact solutions of geophysical fluid dynamics equations for testing models in spherical and plane geometry. $Q$. J. R. Meteorol. Soc. 133: 1605-1614.

Staniforth A, White AA. 2008a. Stability of some exact solutions of the shallowwater equations for testing numerical models in spherical geometry. Q. J. R. Meteorol. Soc. 134: 771-778.

Staniforth A, White AA. 2008b. Unsteady exact solutions of the flow equations for three-dimensional spherical atmospheres. Q. J. R. Meteorol. Soc. 134: $1615-1626$.

Staniforth A, White AA. 2011. Further non-separable exact solutions of the deep- and shallow-atmosphere equations. Atmos. Sci. Lett. 12: 356-361. 
Staniforth A, Wood N. 2008. Aspects of the dynamical core of a nonhydrostatic, deep-atmosphere, unified weather and climate-prediction model. J. Comput. Phys. 227: 3445-3464, doi: 10.1016/j.jcp.2006.11.009.

Staniforth A, Wood N. 2013. Exact axisymmetric solutions of the deep- and shallow-atmosphere Euler equations in curvilinear and plane geometries. Q. J. R. Meteorol. Soc. 139: 1113-1120.

Ullrich PA, Jablonowski C. 2012a. MCore: A non-hydrostatic atmospheric dynamical core utilizing high-order finite-volume methods. J. Comput. Phys. 231: 5078-5108, doi: 10.1016/j.jcp.2012.04.024.

Ullrich PA, Jablonowski C. 2012b. Operator-split Runge-Kutta-Rosenbrock methods for non-hydrostatic atmospheric models. Mon. Weather Rev. 140: $1257-1284$.

Walko RL, Avissar R. 2008. The Ocean-Land-Atmosphere Model (OLAM). Part II: Formulation and tests of the nonhydrostatic dynamic core. Mon. Weather Rev. 136: 4045, doi: 10.1175/2008MWR2523.1.

Wedi N, Smolarkiewicz P. 2009. A framework for testing global non-hydrostatic models. Q. J. R. Meteorol. Soc. 135: 469-484.

White AA, Staniforth A. 2008. A generalized thermal wind equation and some non-separable exact solutions of the flow equations for three-dimensional spherical atmospheres. Q. J. R. Meteorol. Soc. 134: 1931-1939.
White AA, Staniforth A. 2009. Stability criteria for shallow water flow above zonally symmetric orography on the sphere. Q. J. R. Meteorol. Soc. 135: 1897-1905.

White AA, Wood N. 2012. Consistent approximate models of the global atmosphere in non-spherical geopotential coordinates. Q. J. R. Meteorol. Soc. 138: 980-988.

White AA, Hoskins BJ, Roulstone I, Staniforth A. 2005. Consistent approximate models of the global atmosphere: shallow, deep, hydrostatic, quasihydrostatic and non-hydrostatic. Q. J. R. Meteorol. Soc. 131: 2081-2107.

Williamson DL, Drake JB, Hack JJ, Jakob R, Swarztrauber PN. 1992. A standard test set for numerical approximations to the shallow-water equations in spherical geometry. J. Comput. Phys. 102: 211-224.

Williamson DL, Olson JG, Jablonowski C. 2009. Two dynamical core formulation flaws exposed by a baroclinic instability test case. Mon. Weather Rev. 137: 790-796.

Wood N, Staniforth A, White AA, Allen T, Diamantakis M, Gross M, Melvin T, Smith C, Vosper S, Zerroukat M, Thuburn J. 2013. An inherently mass-conserving semi-implicit semi-Lagrangian discretisation of the deepatmosphere global non-hydrostatic equations. Q. J. R. Meteorol. Soc., doi: $10.1002 / q j .2235$. 